\title{
Palladium-Catalyzed Homocoupling and Cross-Coupling Reactions of Aryl Halides in Poly(ethylene glycol)
}

\author{
Liang Wang, Yuhong Zhang*, Leifang Liu, Yanguang Wang* \\ Department of Chemistry, Zhejiang University, Hangzhou 310027, P. R. China. \\ Fax: +86-571-87951895; Tel:+86-571-87953253 \\ E-mail: yhzhang@zjuem.zju.edu.cn;
}

\section{Supporting Information}

\section{Content}

General procedure for the coupling reaction.......................S2

Characterization data of the product of the coupling reaction............S3

References....................................................... 35 
General Starting materials and solvents were purchased from common commercial sources and were used without additional purification. The gas chromatography analysis was performed on a GC instrument. ${ }^{1} \mathrm{H}$ NMR spectra were recorded at 500 MHz, using TMS as internal standard. Mass spectroscopy data of the product of coupling reaction was collected on a MS-EI instrument.

Representative Procedure for the coupling reaction. A mixture of aryl iodides (4 mmol), $\mathrm{Pd}(\mathrm{OAc})_{2}(9 \mathrm{mg}, 0.04 \mathrm{mmol}, 2 \mathrm{~mol} \%), \mathrm{K}_{2} \mathrm{CO}_{3}(0.55 \mathrm{~g}, 4 \mathrm{mmol})$, and PEG 4000 (4 g) were stirred at $120{ }^{\circ} \mathrm{C}$ for the desired time until complete consumption of the starting material as judged by TLC. For aryl bomides, the catalyst of $\mathrm{Pd}(\mathrm{OAc})_{2}$ was increased to $5 \mathrm{~mol} \%$ ( $22 \mathrm{mg}, 0.1 \mathrm{mmol})$. After the reaction the solution was cooled to room temperature and the resulting solid was extracted with anhydrous diethyl ether (4 $\times 15 \mathrm{~mL}$ ). The residue was subjected to a second run with the same substrates without further addition of $\mathrm{Pd}(\mathrm{OAc})_{2}$ and PEG. The combined diethyl ether phase was analyzed by GC/MS and then concentrated. Further purification of the product was achieved by flash chromatography on a silica gel column. All of the prepared compounds are known and the CA numbers as well as the related preparation and characterization reference are presented. 


\section{Characterization data of the product of the Coupling reaction}

T1-1, Biphenyl [92-52-4, ref 1]

${ }^{1} \mathrm{H}$ NMR (500 MHz, $\left.\mathrm{CDCl}_{3}, \mathrm{TMS}\right) \delta 7.60(\mathrm{~d}, 4 \mathrm{H}, J=7.5 \mathrm{~Hz}), 7.44(\mathrm{t}, 4 \mathrm{H}, J=7.2 \mathrm{~Hz})$,

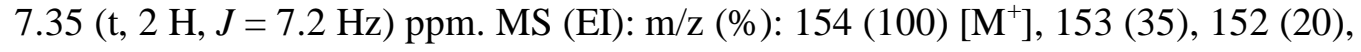

115 (3), 76 (13), 51 (4).
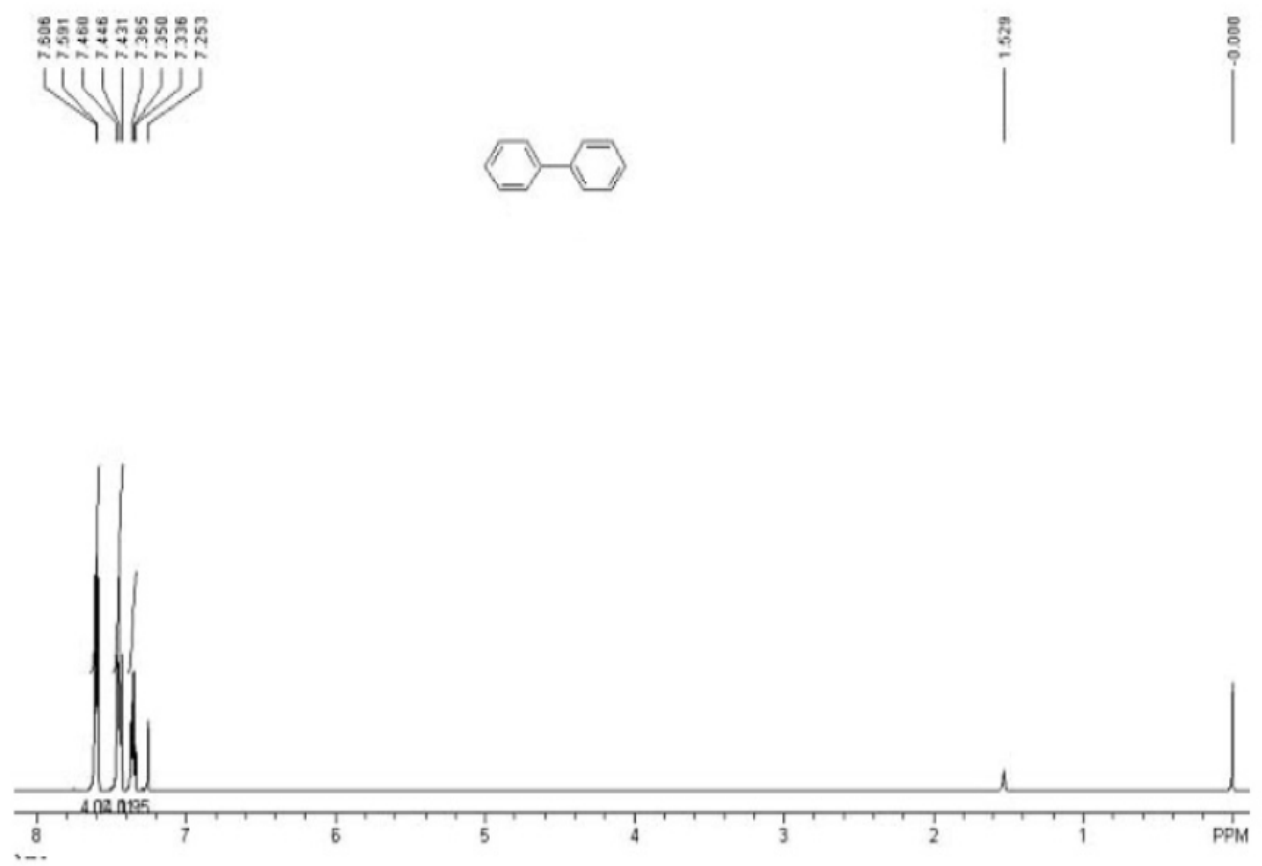


\section{T 2-1, 4,4'-dinitro-biphenyl [1528-74-1, ref 2]}

${ }^{1} \mathrm{H}$ NMR (500 MHz, $\mathrm{CDCl}_{3}$, TMS) $\delta 8.36(\mathrm{~d}, 4 \mathrm{H}, J=9.0 \mathrm{~Hz}), 7.79(\mathrm{~d}, 4 \mathrm{H}, J=9.0 \mathrm{~Hz})$

ppm. MS (EI): m/z (\%): 244 (100) [M+], 198 (15), 152 (31), 121 (11), 76 (7), 46 (2).
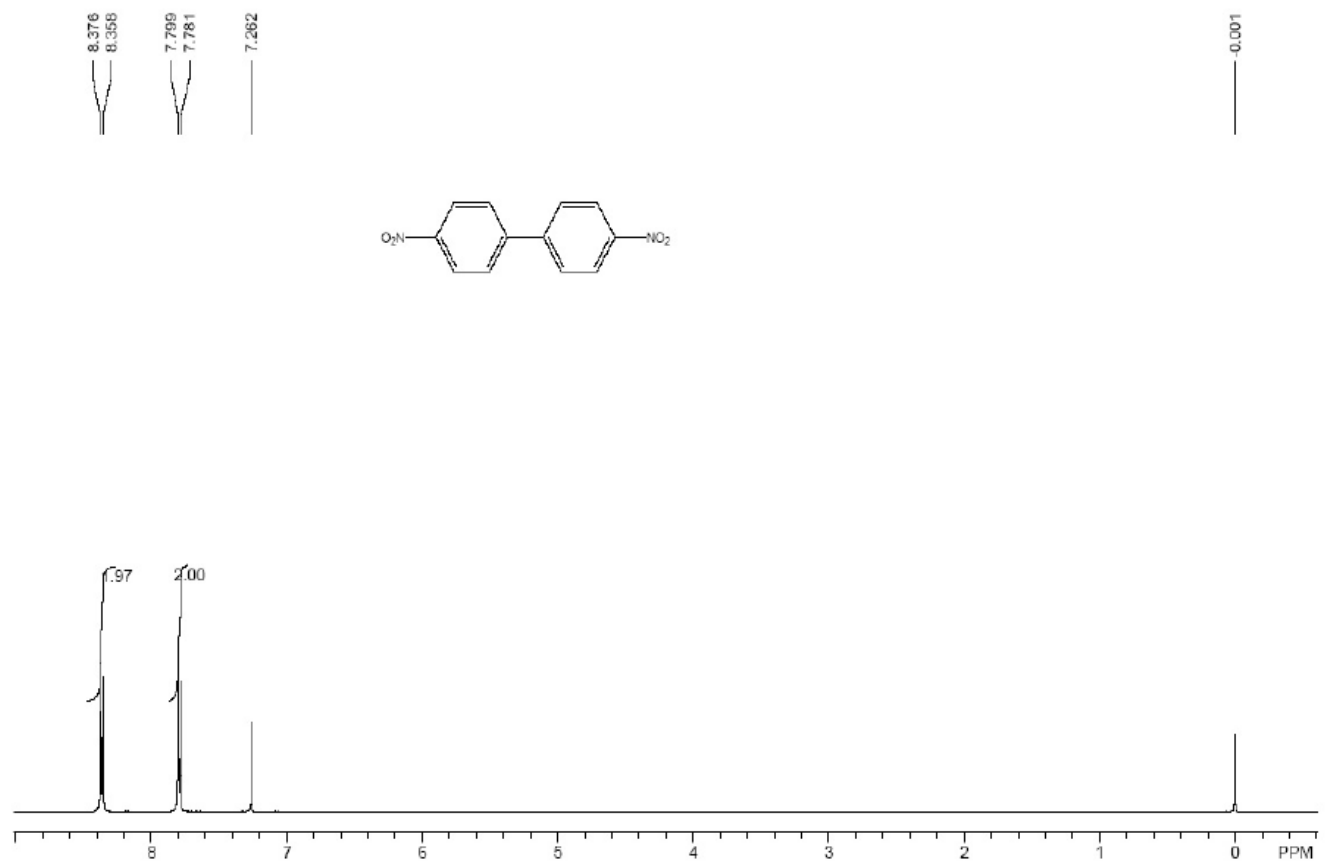
T 2-2, Biphenyl-4,4'-dicarboxylic acid dimethyl ester [47230-38-6, ref 1]

${ }^{1} \mathrm{H}$ NMR $\left(500 \mathrm{MHz}, \mathrm{CDCl}_{3}, \mathrm{TMS}\right) \delta 8.13(\mathrm{~d}, 4 \mathrm{H}, J=8.5 \mathrm{~Hz}), 7.68(\mathrm{~d}, 4 \mathrm{H}, J=8.5 \mathrm{~Hz})$,

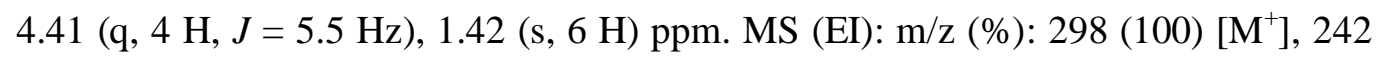

(15), 152 (32), $76(7)$.

4
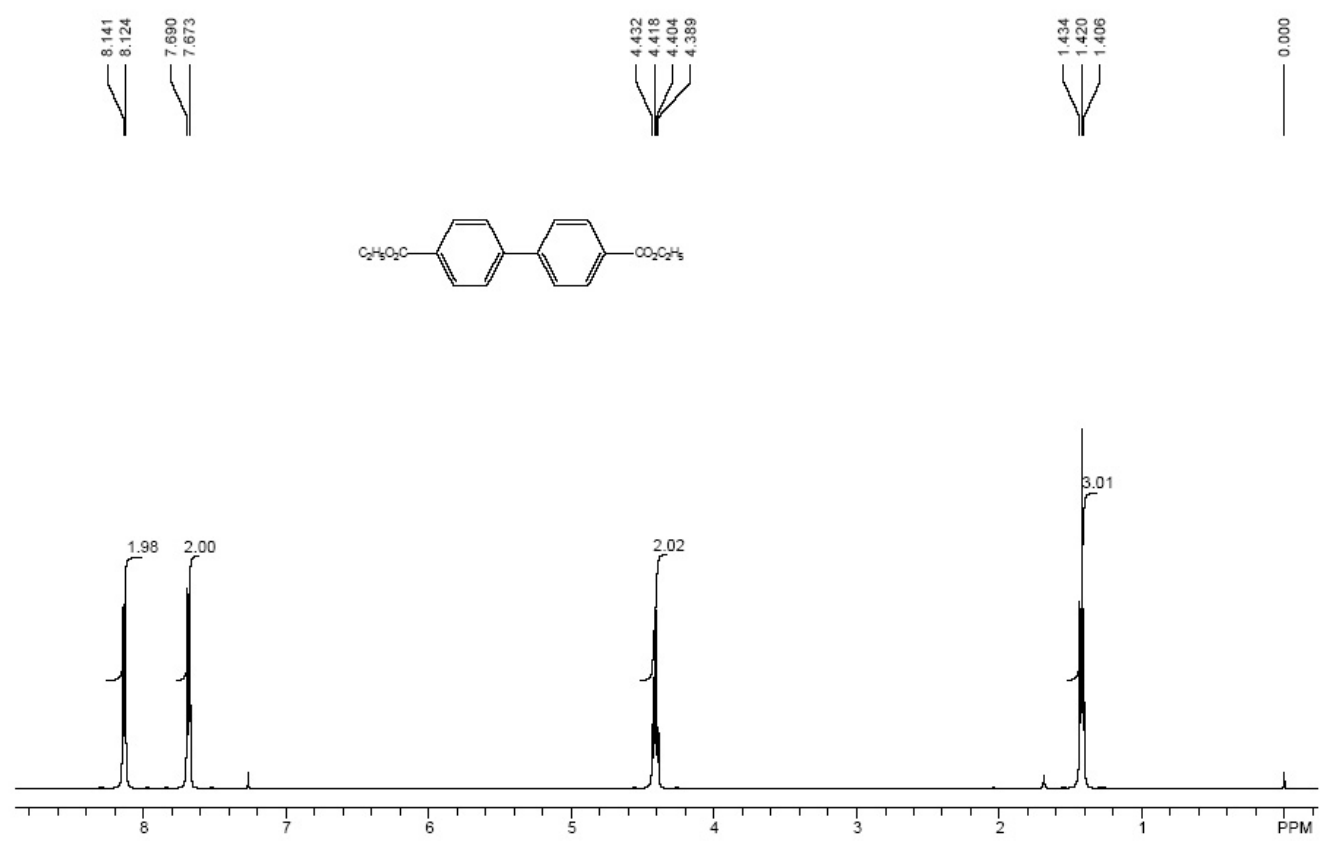
T 2-3, Biphenyl-3,3'-dicarboxylic acid diethyl ester [1751-97-9, ref 3]

${ }^{1} \mathrm{H}$ NMR (500 MHz, $\left.\mathrm{CDCl}_{3}, \mathrm{TMS}\right) \delta 8.30(\mathrm{~s}, 2 \mathrm{H}), 8.05(\mathrm{~d}, 2 \mathrm{H}, J=7.5 \mathrm{~Hz}), 7.81(\mathrm{~d}, 2$

$\mathrm{H}, J=7.5 \mathrm{~Hz}), 7.54(\mathrm{t}, 2 \mathrm{H}, J=5.0 \mathrm{~Hz}), 3.96$ (s, $6 \mathrm{H}) \mathrm{ppm} . \mathrm{MS}(\mathrm{EI}): \mathrm{m} / \mathrm{z}(\%): 270(95)$

$\left[\mathrm{M}^{+}\right], 239$ (100), $152(45), 104(21), 76(20)$.

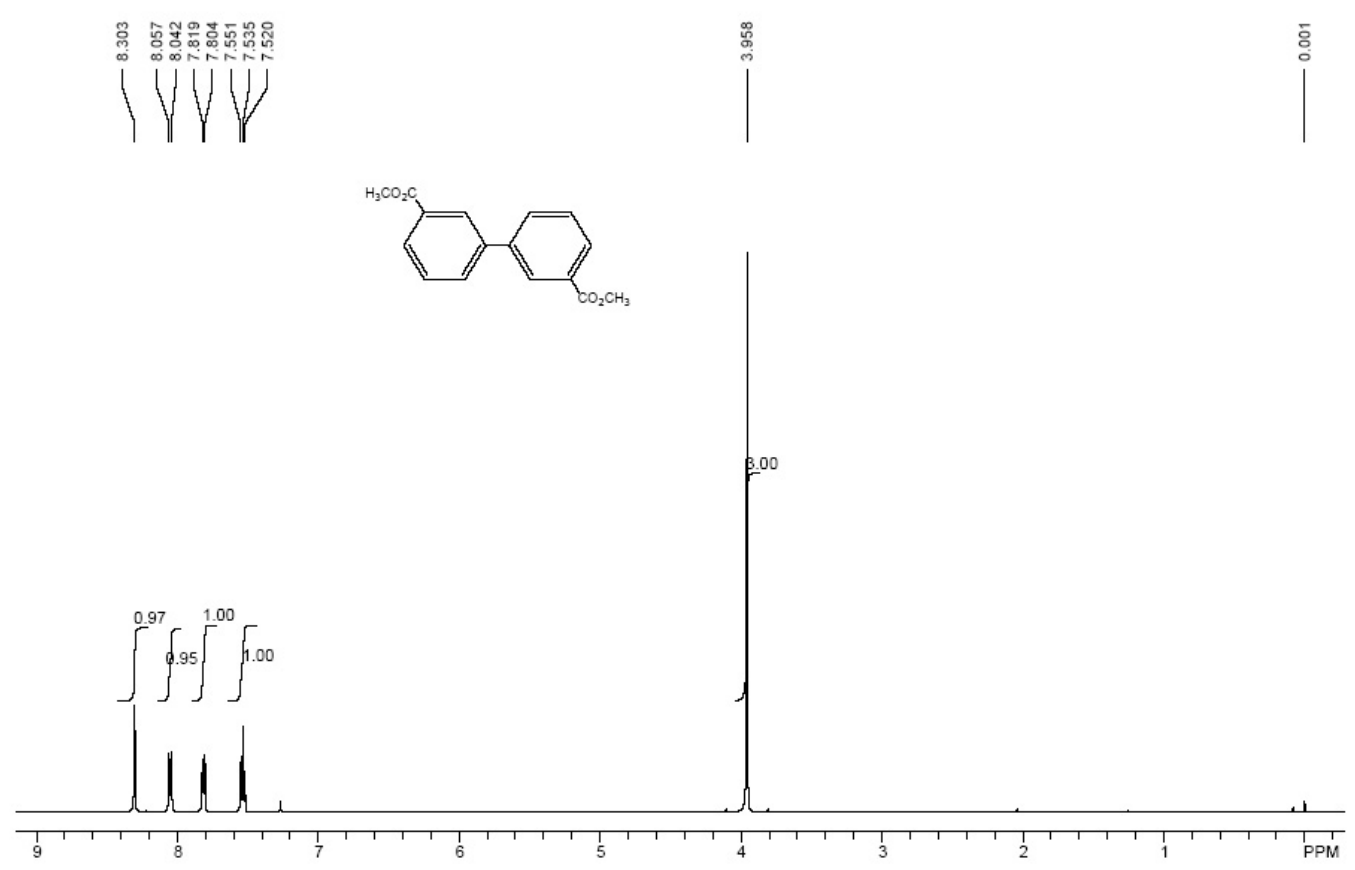


T 2-4, Biphenyl-2,2'-dicarboxylic acid diethyl ester [5807-64-7, ref 4]

${ }^{1} \mathrm{H}$ NMR (500 MHz, $\left.\mathrm{CDCl}_{3}, \mathrm{TMS}\right) \delta 8.00(\mathrm{q}, 2 \mathrm{H}, J=2.5 \mathrm{~Hz}), 7.52(\mathrm{t}, 2 \mathrm{H}, J=3.0 \mathrm{~Hz})$,

$7.43(\mathrm{t}, 2 \mathrm{H}, J=3.0 \mathrm{~Hz}), 7.21(\mathrm{q}, 2 \mathrm{H}, J=2.0 \mathrm{~Hz}), 3.61(\mathrm{~s}, 6 \mathrm{H}) \mathrm{ppm} . \mathrm{MS}(\mathrm{EI}): \mathrm{m} / \mathrm{z}(\%)$ :

$270(7)\left[\mathrm{M}^{+}\right], 211$ (100), $196(20), 152$ (18), 139 (15), 76 (14).
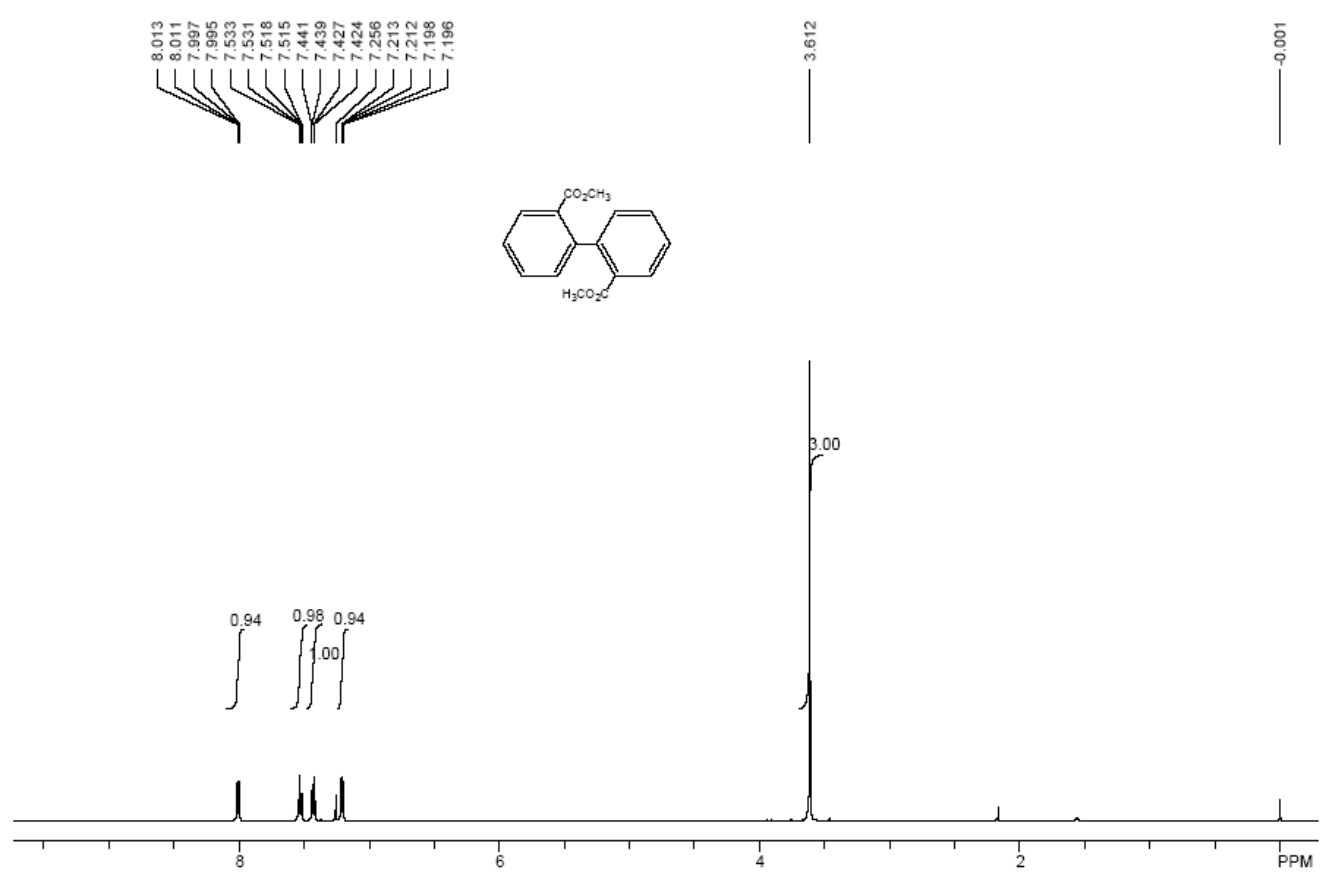


\section{T 2-5, 4,4'-dichloro-biphenyl [37677-93-3, ref 1]}

${ }^{1} \mathrm{H}$ NMR (500 MHz, $\mathrm{CDCl}_{3}$, TMS) $\delta 7.48(\mathrm{~d}, 4 \mathrm{H}, J=8.0 \mathrm{~Hz}), 7.40(\mathrm{~d}, 4 \mathrm{H}, J=8.0 \mathrm{~Hz})$

ppm. MS (EI): m/z (\%): 222 (100) [M+], 186 (15), 152 (51), 111 (5), 93 (7), 75 (9).
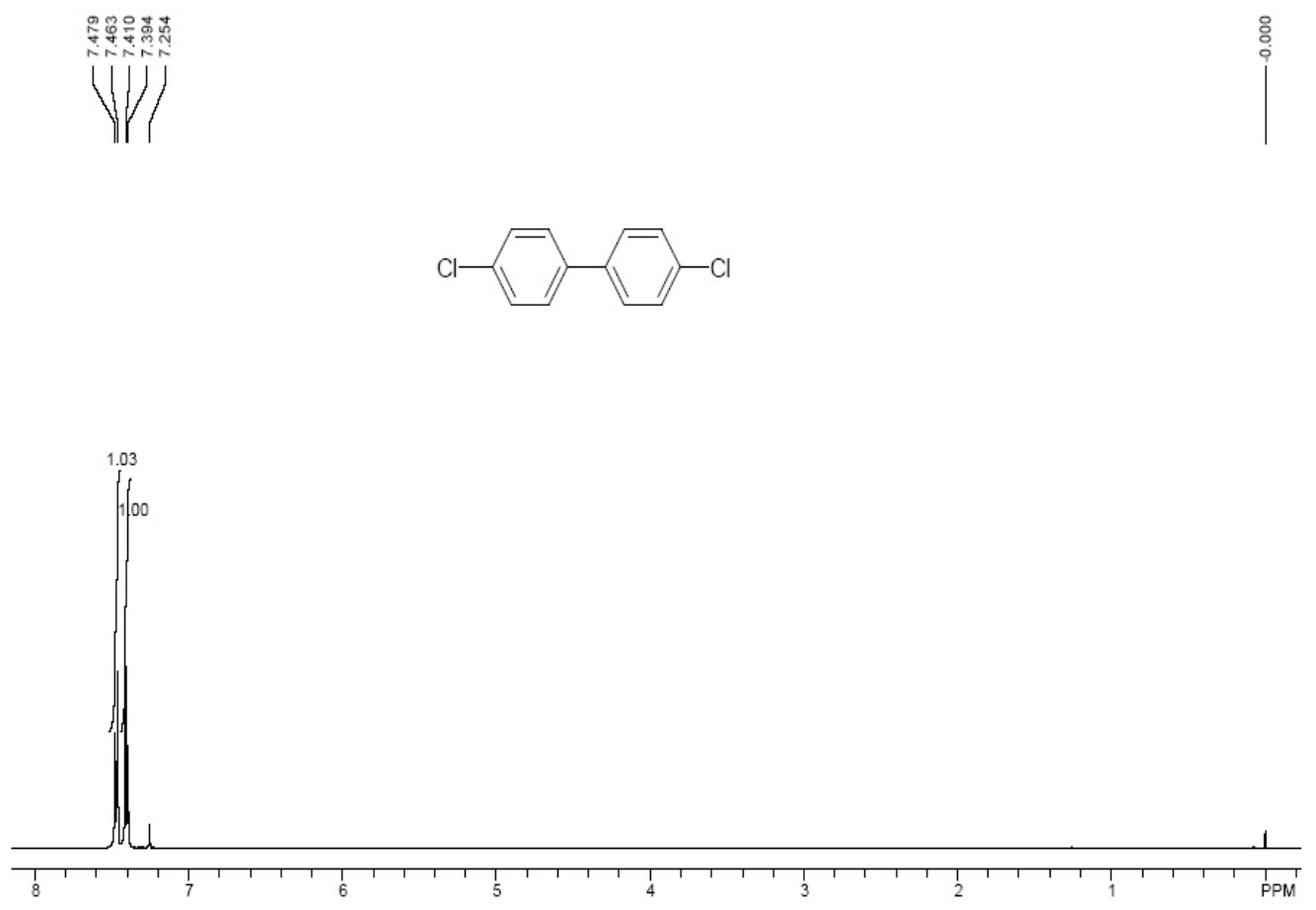


\section{T 2-7, 4,4'-dimethyl-biphenyl [613-33-2, ref 2]}

${ }^{1} \mathrm{H}$ NMR (500 MHz, $\left.\mathrm{CDCl}_{3}, \mathrm{TMS}\right) \delta 7.66(\mathrm{~d}, 4 \mathrm{H}, J=8.0 \mathrm{~Hz}), 7.41(\mathrm{~d}, 4 \mathrm{H}, J=8.0 \mathrm{~Hz})$,

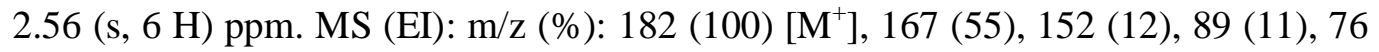
(3).
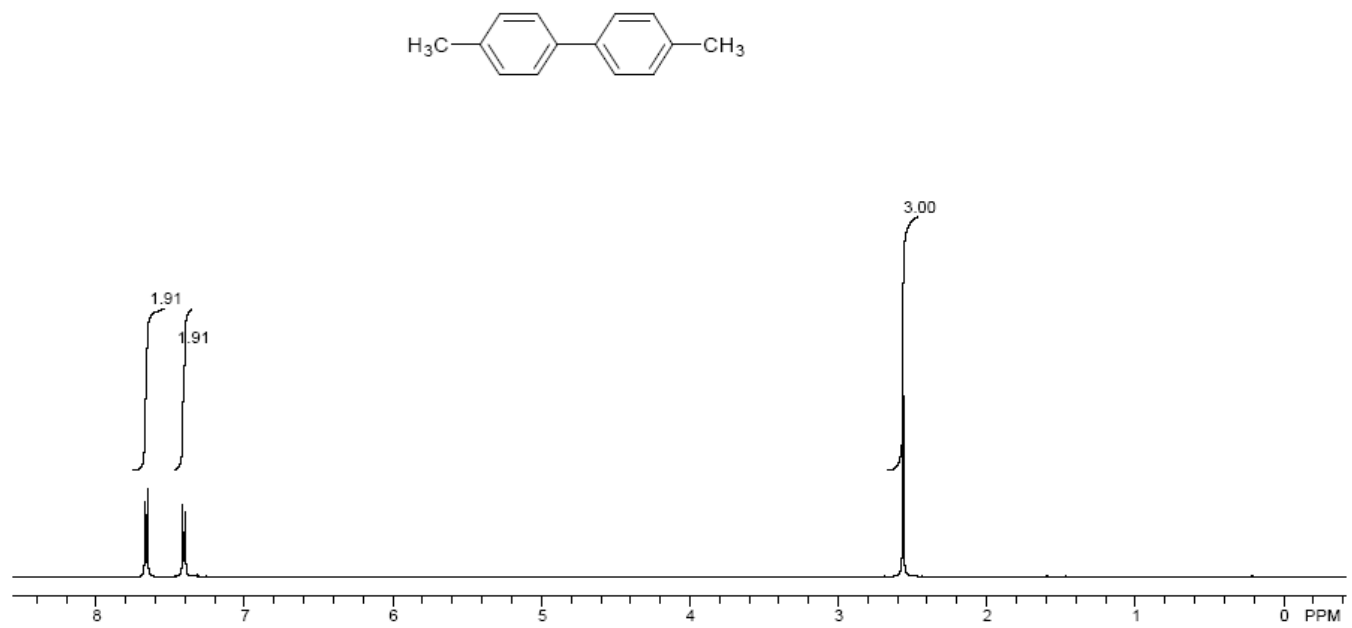
T 2-8, 4,4'-dimethoxy-biphenyl [2132-80-1, ref 2]

${ }^{1} \mathrm{H} \mathrm{NMR}\left(500 \mathrm{MHz}, \mathrm{CDCl}_{3}, \mathrm{TMS}\right) \delta 7.47(\mathrm{~d}, 4 \mathrm{H}, J=9.0 \mathrm{~Hz}), 6.95(\mathrm{~d}, 4 \mathrm{H}, J=9.0 \mathrm{~Hz})$,

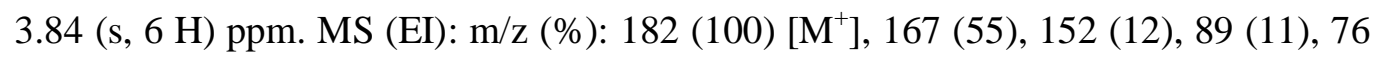
(3).
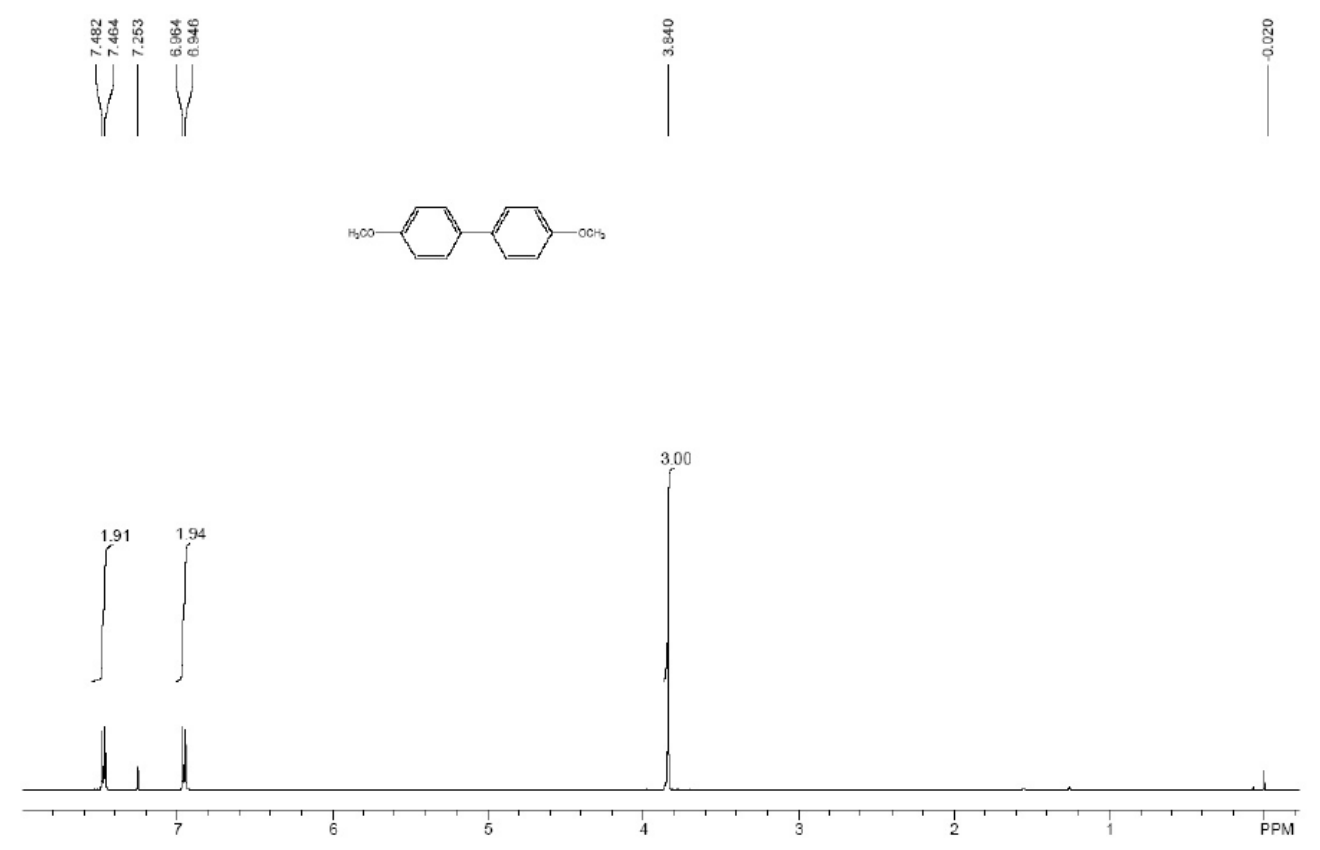


\section{T 2-10, 3,3'-dinitro-biphenyl [958-96-3, ref 5]}

${ }^{1} \mathrm{H}$ NMR (500 MHz, $\mathrm{CDCl}_{3}$, TMS) $\delta 8.50(\mathrm{t}, 2 \mathrm{H}, J=2.0 \mathrm{~Hz}), 8.30(\mathrm{~m}, 2 \mathrm{H}), 7.97(\mathrm{~m}, 2$

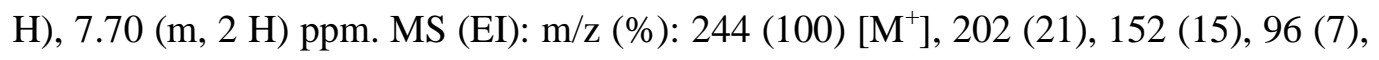
$76(15), 62(5)$.
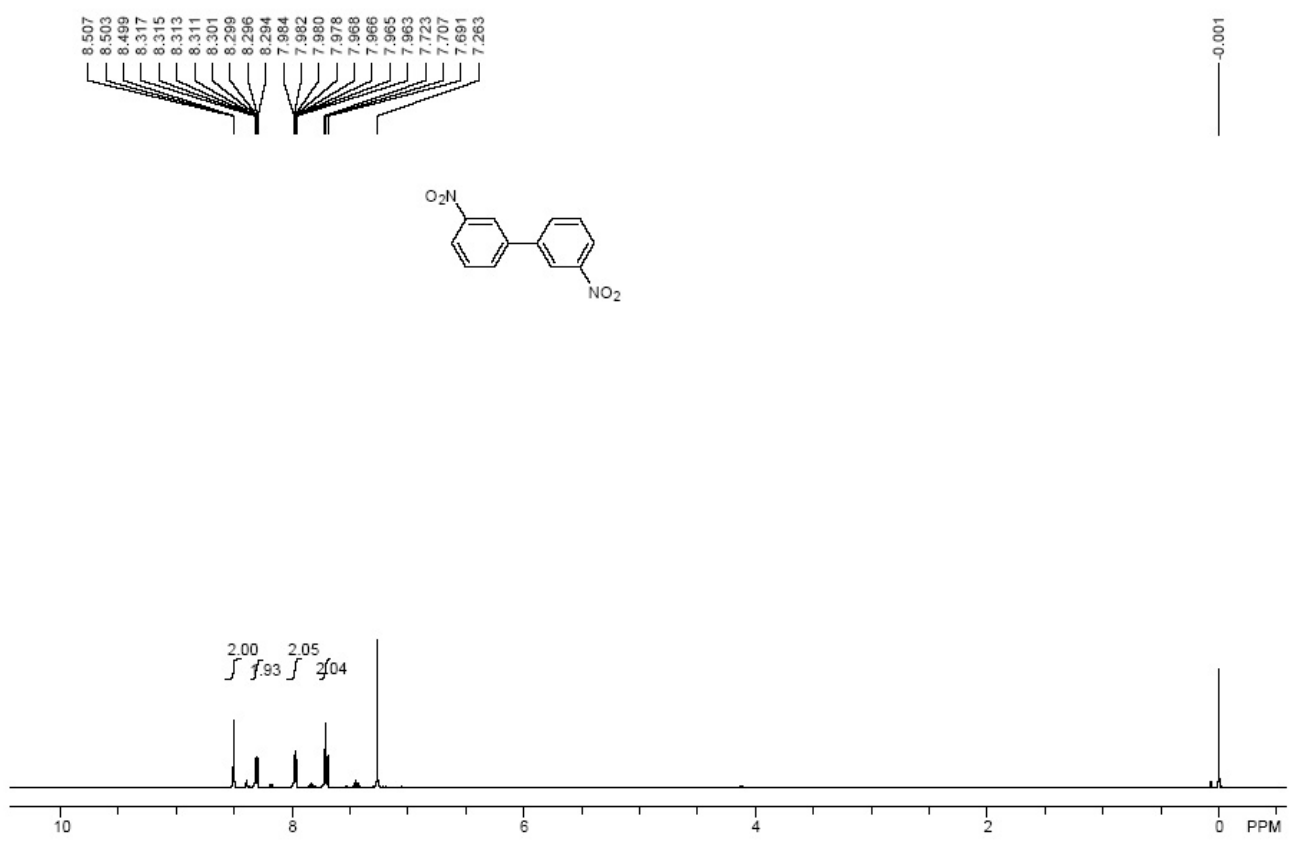


\section{T 2-11, 4,4'-dicyano-biphenyl [1591-30-6, ref 2]}

${ }^{1} \mathrm{H}$ NMR (500 MHz, $\mathrm{CDCl}_{3}$, TMS) $\delta 7.78(\mathrm{~d}, 4 \mathrm{H}, J=6.0 \mathrm{~Hz}), 7.70(\mathrm{~d}, 2 \mathrm{H}, J=6.5 \mathrm{~Hz})$ ppm. MS (EI): m/z (\%): 204 (100) [M+], 180 (9), 152 (23), 92 (5), 76 (8).
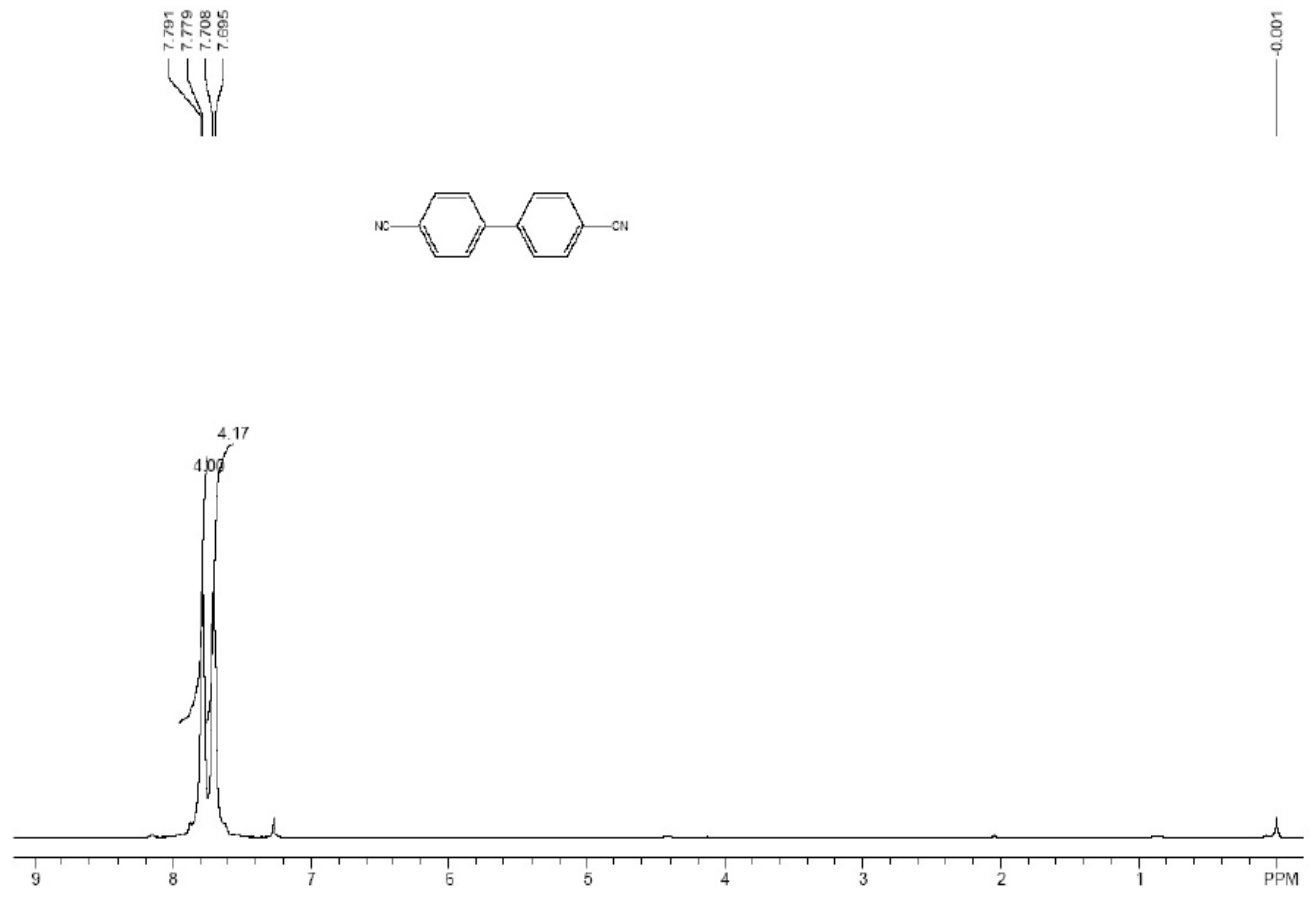


\section{T 2-12, 3,3'-dicyano-biphenyl [34488-69-2, ref 6]}

${ }^{1} \mathrm{H}$ NMR (500 MHz, $\mathrm{CDCl}_{3}$, TMS) $\delta 7.78(\mathrm{~d}, 4 \mathrm{H}, J=6.0 \mathrm{~Hz}), 7.70(\mathrm{~d}, 2 \mathrm{H}, J=6.5 \mathrm{~Hz})$ ppm. MS (EI): m/z (\%): 204 (100) $\left[\mathrm{M}^{+}\right], 180$ (9), 152 (23), 92 (5), 76 (8).
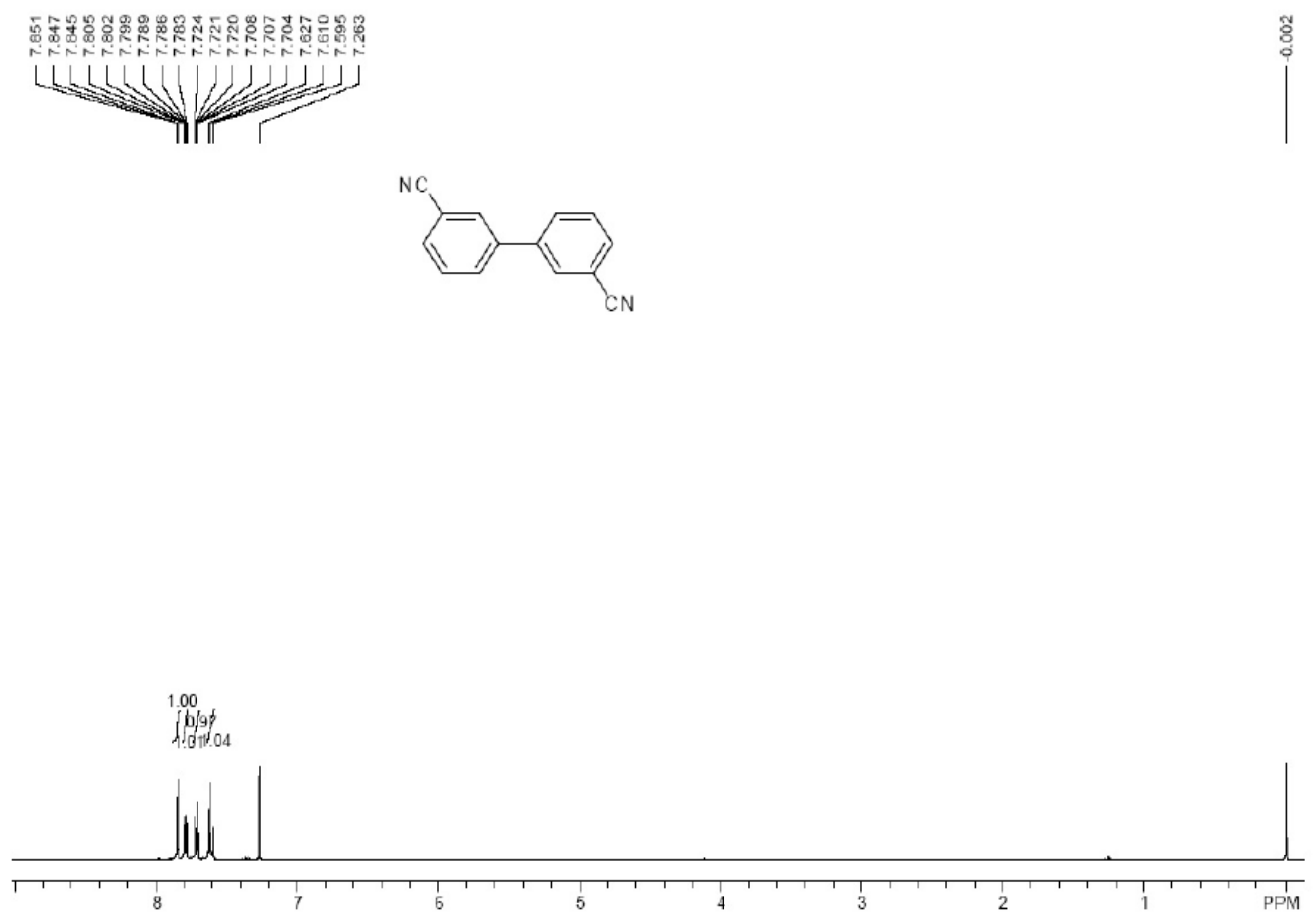


\section{T 2-13, 4,4'-diacetyl-biphenyl [787-69-9, ref 1]}

${ }^{1} \mathrm{H}$ NMR $\left(500 \mathrm{MHz}, \mathrm{CDCl}_{3}, \mathrm{TMS}\right) \delta 8.06(\mathrm{~d}, 4 \mathrm{H}, J=8.5 \mathrm{~Hz}), 7.72(\mathrm{~d}, 4 \mathrm{H}, J=8.5 \mathrm{~Hz})$,

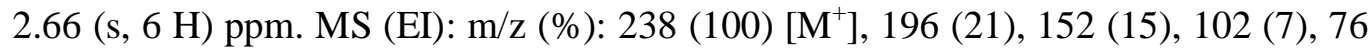
(11).
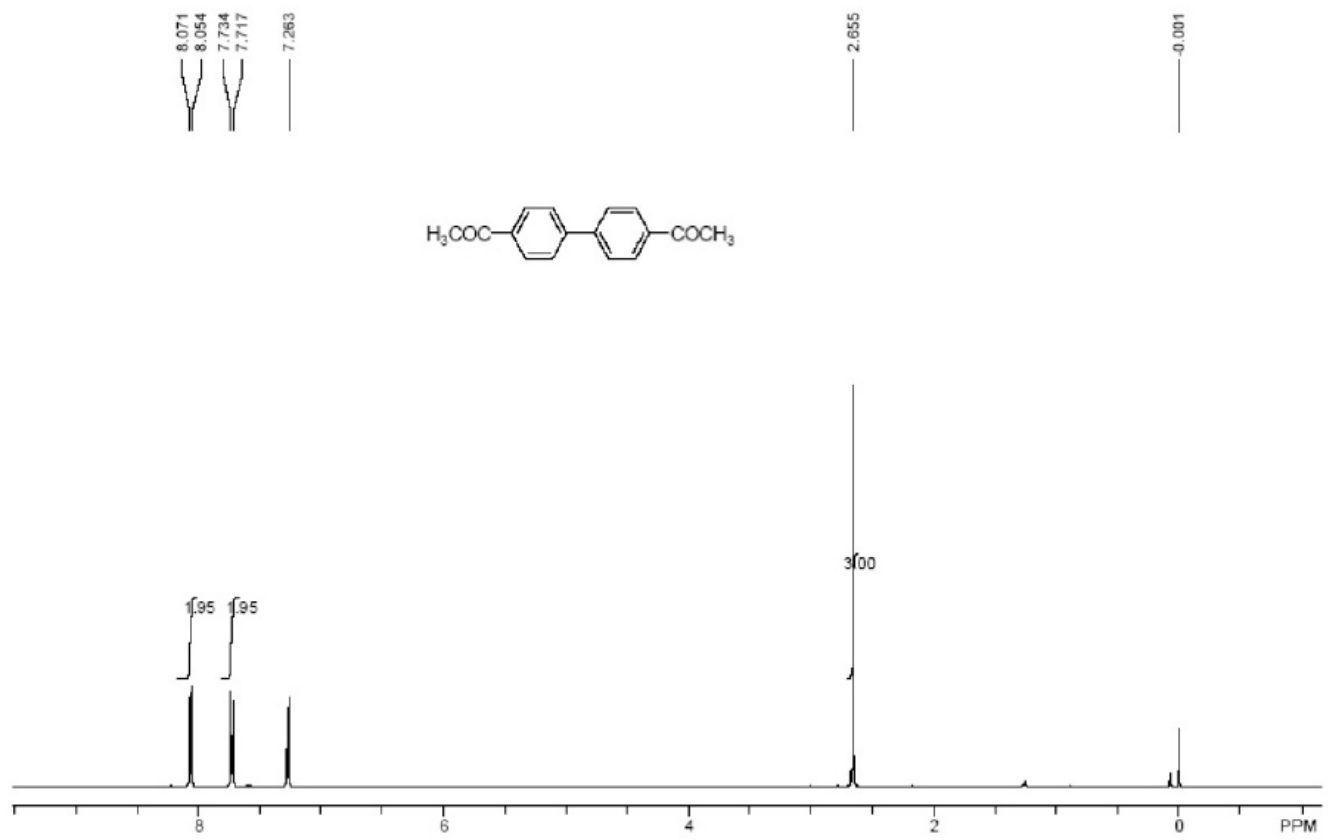


\section{T 2-18, 2,2'Bipyridinyl [366-18-7, ref 1]}

${ }^{1} \mathrm{H} \mathrm{NMR}\left(500 \mathrm{MHz}, \mathrm{CDCl}_{3}, \mathrm{TMS}\right) \delta 8.67$ (q, $\left.2 \mathrm{H}, J=1.5 \mathrm{~Hz}\right), 8.39(\mathrm{q}, 2 \mathrm{H}, J=2.5 \mathrm{~Hz})$, $7.81(\mathrm{~m}, 2 \mathrm{H}), 7.30$ (m, $2 \mathrm{H})$ ppm. MS (EI): m/z (\%): $156(100)\left[\mathrm{M}^{+}\right], 154$ (9), 153 (15), $66(15)$.
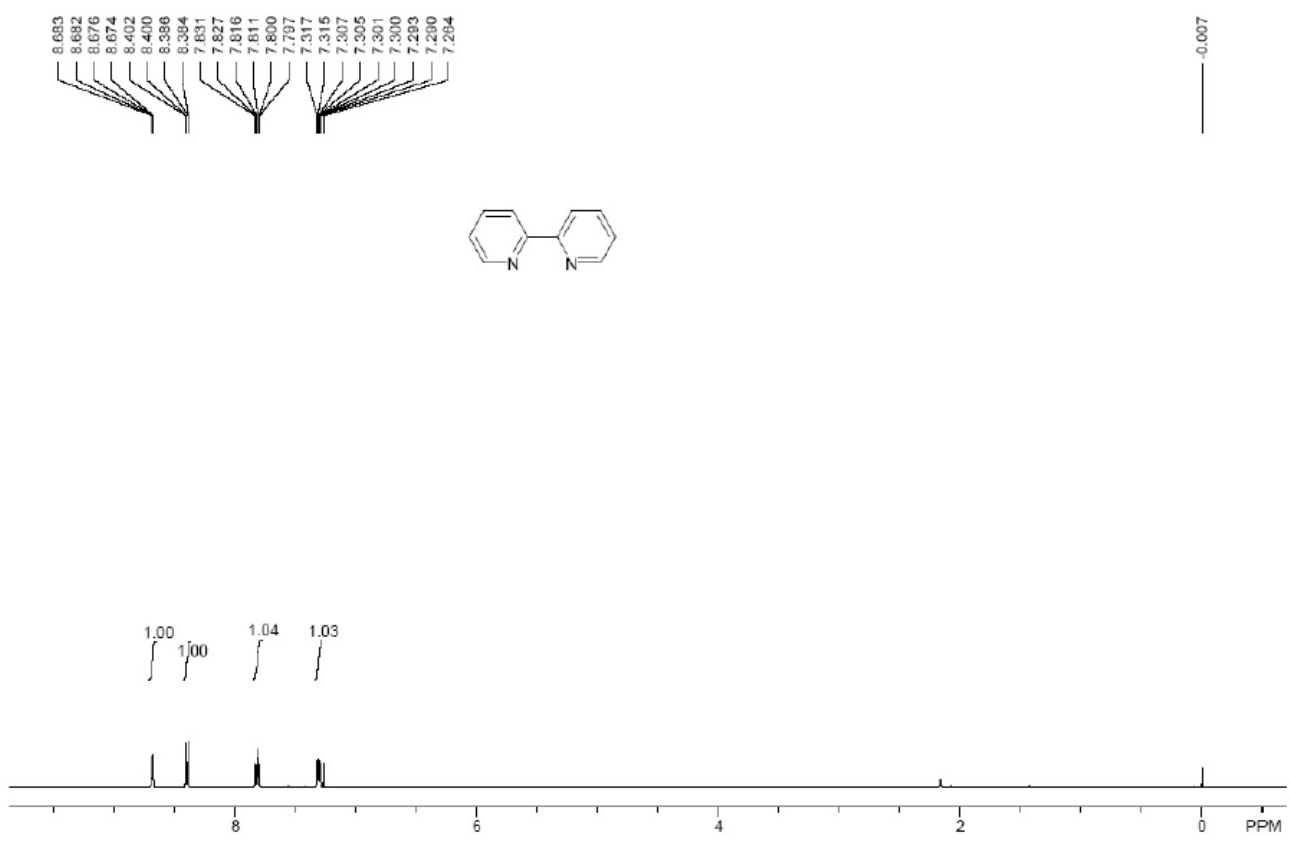


\section{T 2-19, 3,3'Bipyridinyl [581-46-4, ref 1]}

${ }^{1} \mathrm{H}$ NMR (500 MHz, $\mathrm{CDCl}_{3}$, TMS) $\delta 8.76(\mathrm{~s}, 2 \mathrm{H}), 8.57(\mathrm{t}, 2 \mathrm{H}, J=2.5 \mathrm{~Hz}), 7.81(\mathrm{~m}, 2$ H), 7.34 (m, 2 H) ppm. MS (EI): m/z (\%): 156 (100) [M+1, 152 (11), 72 (5), 66 (21).
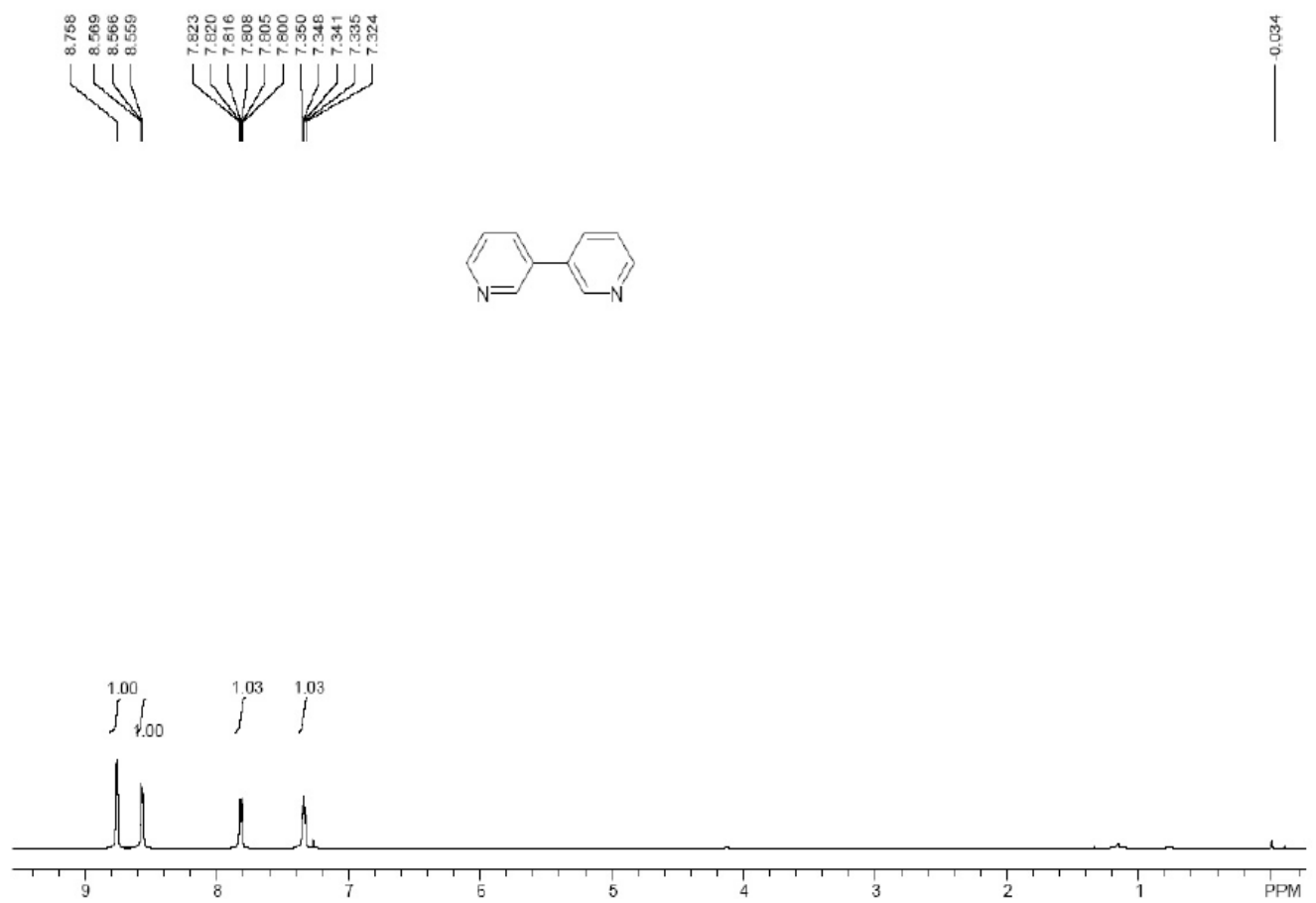
T 2-20, 2,2'Bithiophenyl [492-91-7, ref 1]

${ }^{1} \mathrm{H} \mathrm{NMR}\left(500 \mathrm{MHz}, \mathrm{CDCl}_{3}, \mathrm{TMS}\right) \delta 7.20(\mathrm{~d}, 2 \mathrm{H}, J=5.5 \mathrm{~Hz}), 7.17(\mathrm{~d}, 2 \mathrm{H}, J=3.5 \mathrm{~Hz})$,

7.01 (m, 2 H) ppm. MS (EI): m/z (\%): $166(100)\left[\mathrm{M}^{+}\right], 165$ (9), 164 (5), 82 (15).
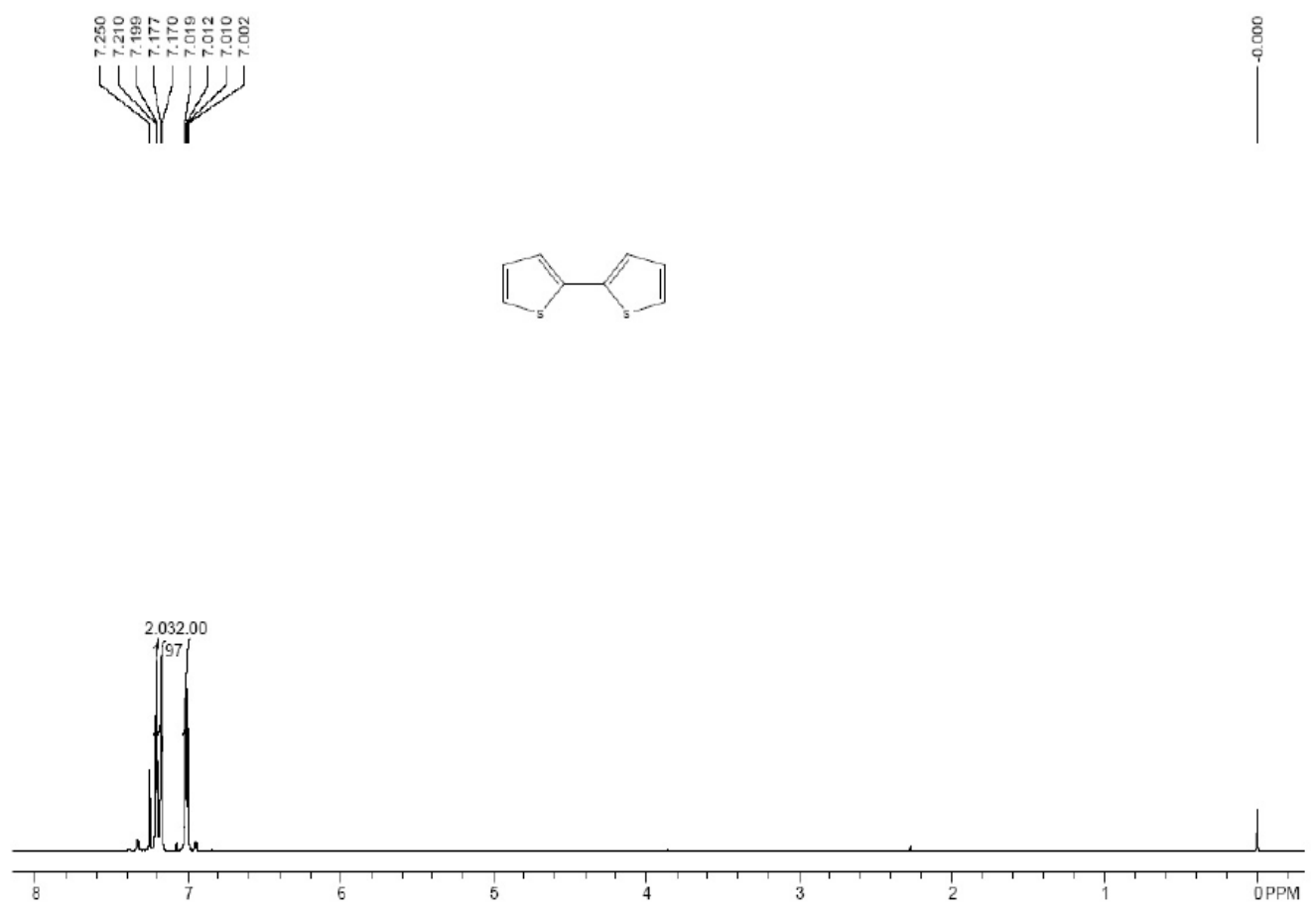


\section{T 2-21, 3,3'Bithiophenyl [3172-56-3, ref 7]}

${ }^{1} \mathrm{H}$ NMR (500 MHz, $\mathrm{CDCl}_{3}$, TMS) $\delta 7.37$ (m, $\left.2 \mathrm{H}\right), 7.33$ (m, $\left.4 \mathrm{H}\right) \mathrm{ppm} . \mathrm{MS}(\mathrm{EI}): \mathrm{m} / \mathrm{z}$ (\%): $166(100)\left[\mathrm{M}^{+}\right], 83(21)$.
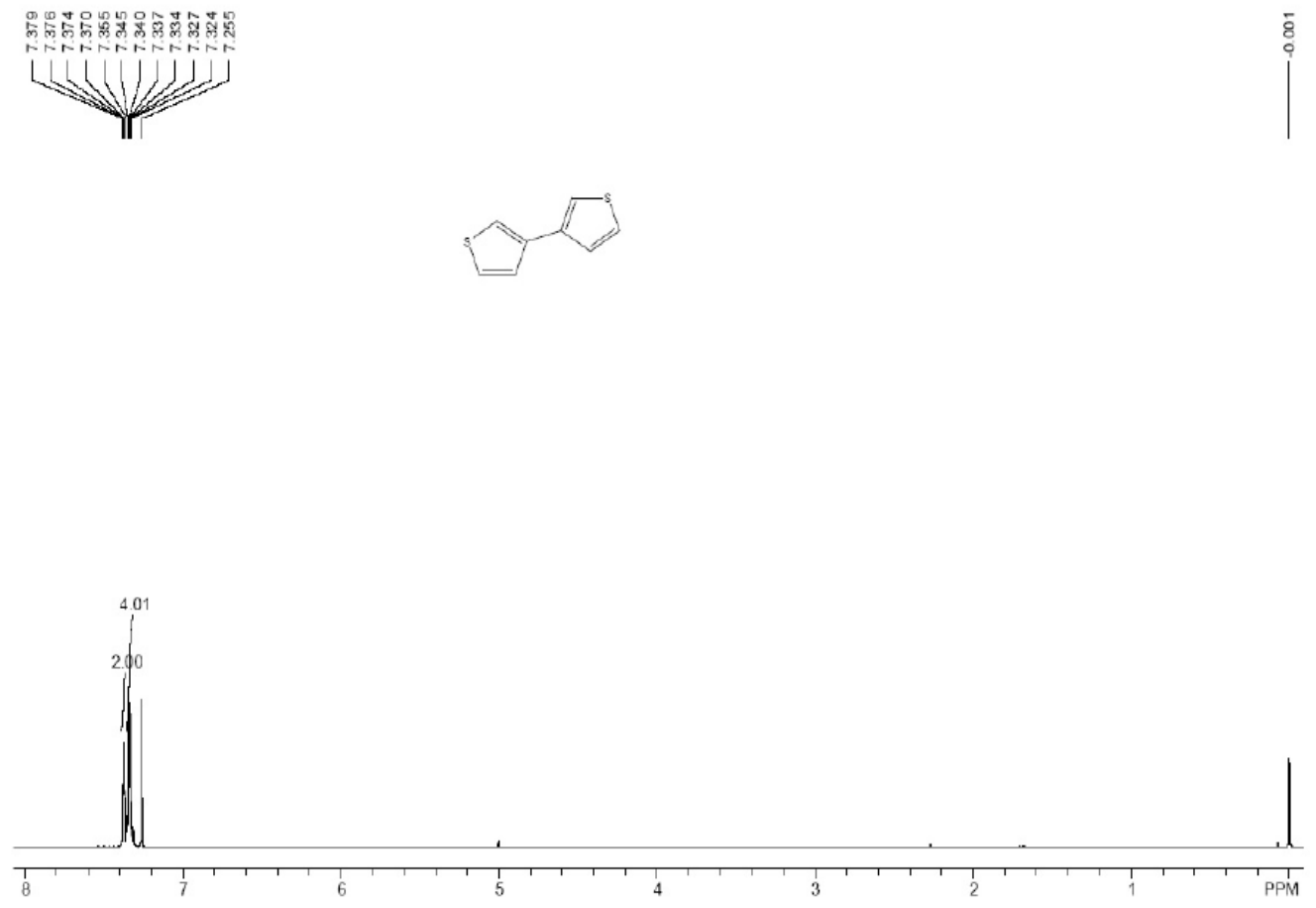
T3-1, Biphenyl-4-carbonitrile [2920-38-9, ref 8]

${ }^{1} \mathrm{H}$ NMR (500 MHz, $\left.\mathrm{CDCl}_{3}, \mathrm{TMS}\right) \delta 7.73(\mathrm{~d}, 2 \mathrm{H}, J=8.5 \mathrm{~Hz}), 7.68(\mathrm{~d}, 2 \mathrm{H}, J=8.5 \mathrm{~Hz})$,

7.59 (t, $2 \mathrm{H}, J=4.5 \mathrm{~Hz}), 7.48(\mathrm{t}, 2 \mathrm{H}, J=7.5 \mathrm{~Hz}), 7.42(\mathrm{~m}, 1 \mathrm{H}) \mathrm{ppm}$. MS (EI): m/z

(\%):179 (100) $\left[\mathrm{M}^{+}\right], 151(16), 89(10), 76(14)$
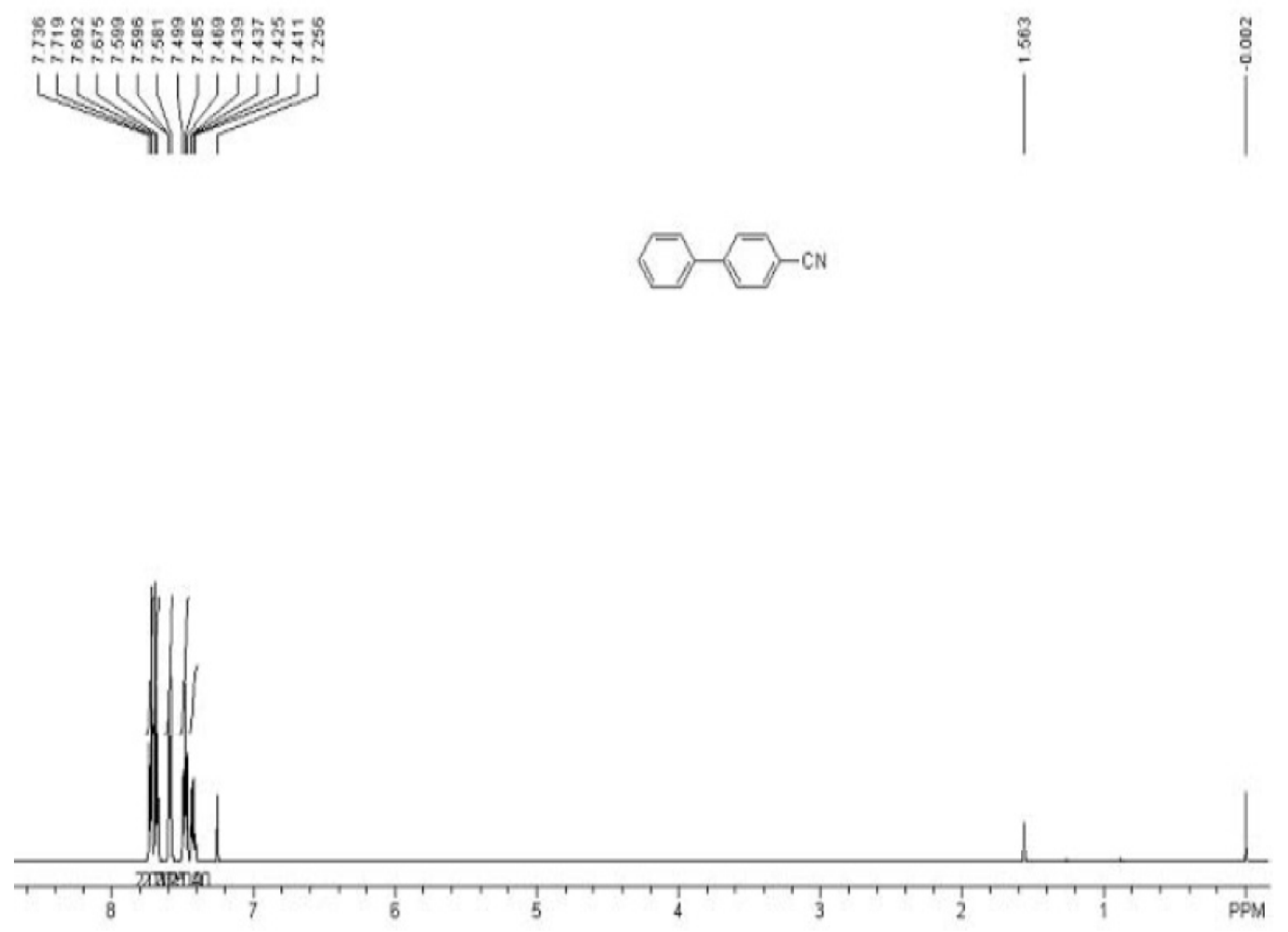
T3-2, 4-Nitro-biphenyl [92-93-3, ref 8]

${ }^{1} \mathrm{H}$ NMR $\left(500 \mathrm{MHz}, \mathrm{CDCl}_{3}, \mathrm{TMS}\right) \delta 8.32(\mathrm{~d}, 2 \mathrm{H}, J=9.0 \mathrm{~Hz}), 7.75(\mathrm{~d}, 2 \mathrm{H}, J=9.0 \mathrm{~Hz})$, $7.64(\mathrm{~d}, 2 \mathrm{H}, J=7.0 \mathrm{~Hz}), 7.51(\mathrm{t}, 2 \mathrm{H}, J=7.5 \mathrm{~Hz}), 7.46(\mathrm{t}, 1 \mathrm{H}, J=7.2 \mathrm{~Hz}) \mathrm{ppm} . \mathrm{MS}$ (EI): m/z (\%): 199 (100) [M+'], 169 (37), 152 (100), 141 (24), 115 (13), 76 (13), 63 (7), $51(6)$
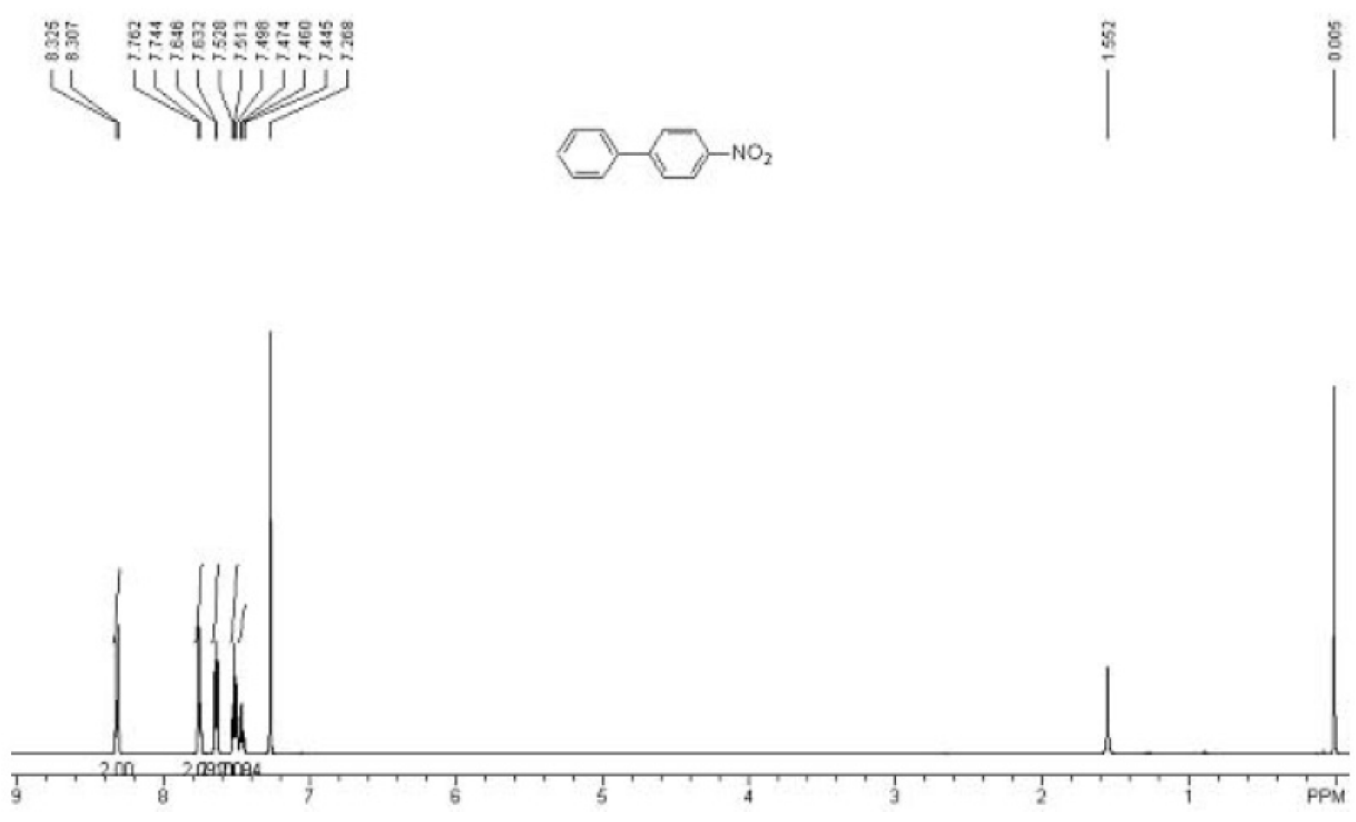
T3-3 4'-Methoxy-4-nitro-biphenyl [2143-90-0, ref 9]

${ }^{1} \mathrm{H}$ NMR (500 MHz, $\mathrm{CDCl}_{3}$, TMS) $\delta 8.28(\mathrm{~d}, 2 \mathrm{H}, J=9.0 \mathrm{~Hz}), 7.71(\mathrm{~d}, 1 \mathrm{H}, J=16.1$

Hz), $7.69(\mathrm{~d}, 2 \mathrm{H}, J=8.5 \mathrm{~Hz}), 7.59(\mathrm{~d}, 2 \mathrm{H}, J=8.5 \mathrm{~Hz}), 7.02(\mathrm{~d}, 2 \mathrm{H}, J=9.0 \mathrm{~Hz}), 3.88$

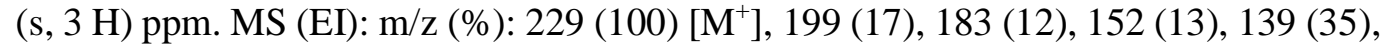

128 (6), 63 (4).
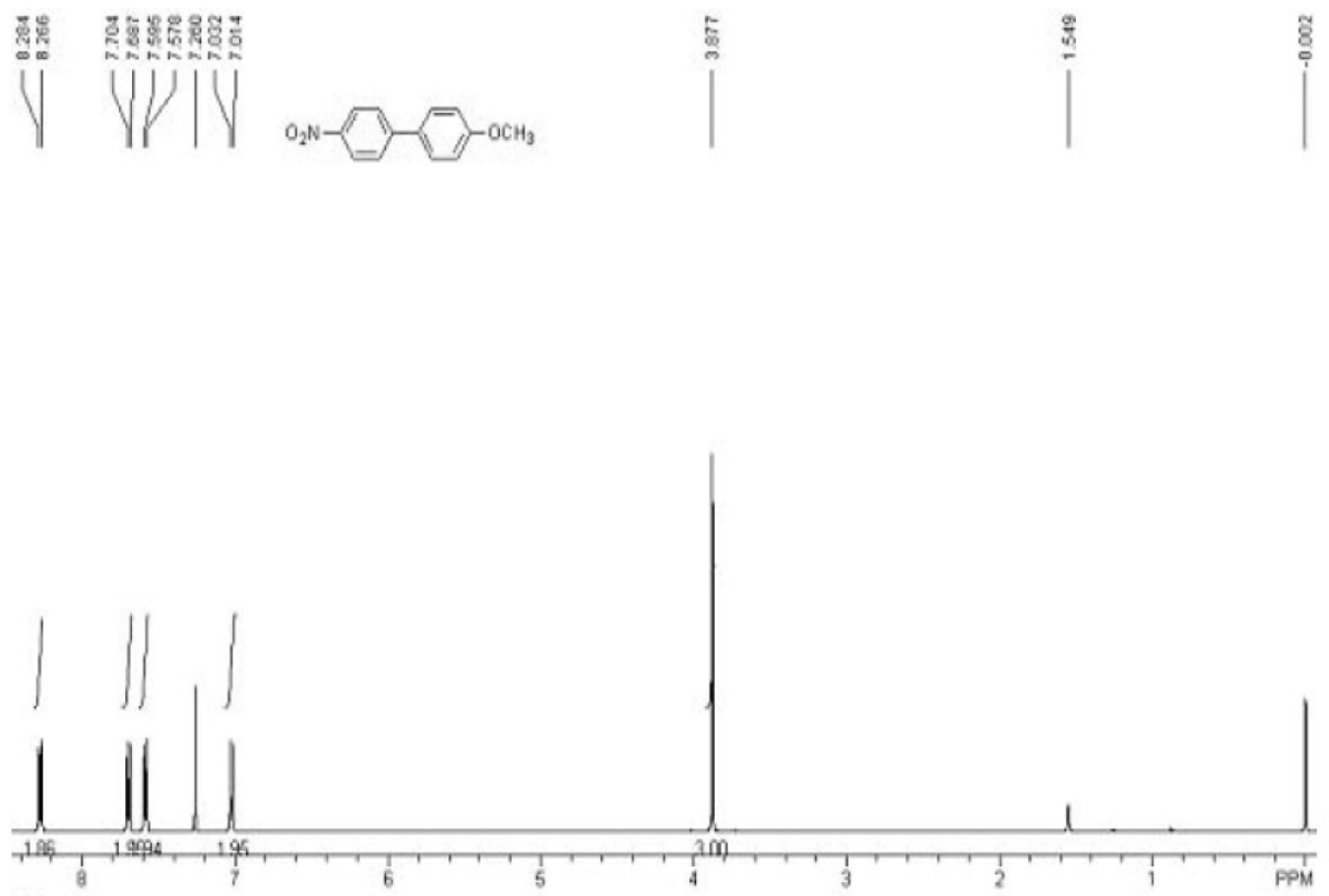
T 3-4 3-Nitro-biphenyl [2113-58-8, ref 10]

${ }^{1} \mathrm{H}$ NMR $\left(500 \mathrm{MHz}, \mathrm{CDCl}_{3}, \mathrm{TMS}\right) \delta 8.45(\mathrm{~s}, 1 \mathrm{H}), 8.19(\mathrm{~m}, 1 \mathrm{H}), 7.91(\mathrm{~d}, 1 \mathrm{H}, J=$

7.5Hz), 7.63 (m, 3 H), 7.49 (m, 2 H), 7.44 (m, 1 H) ppm. MS (EI): m/z (\%): 199 (100)

$\left[\mathrm{M}^{+}\right], 141(15), 115(25)$.
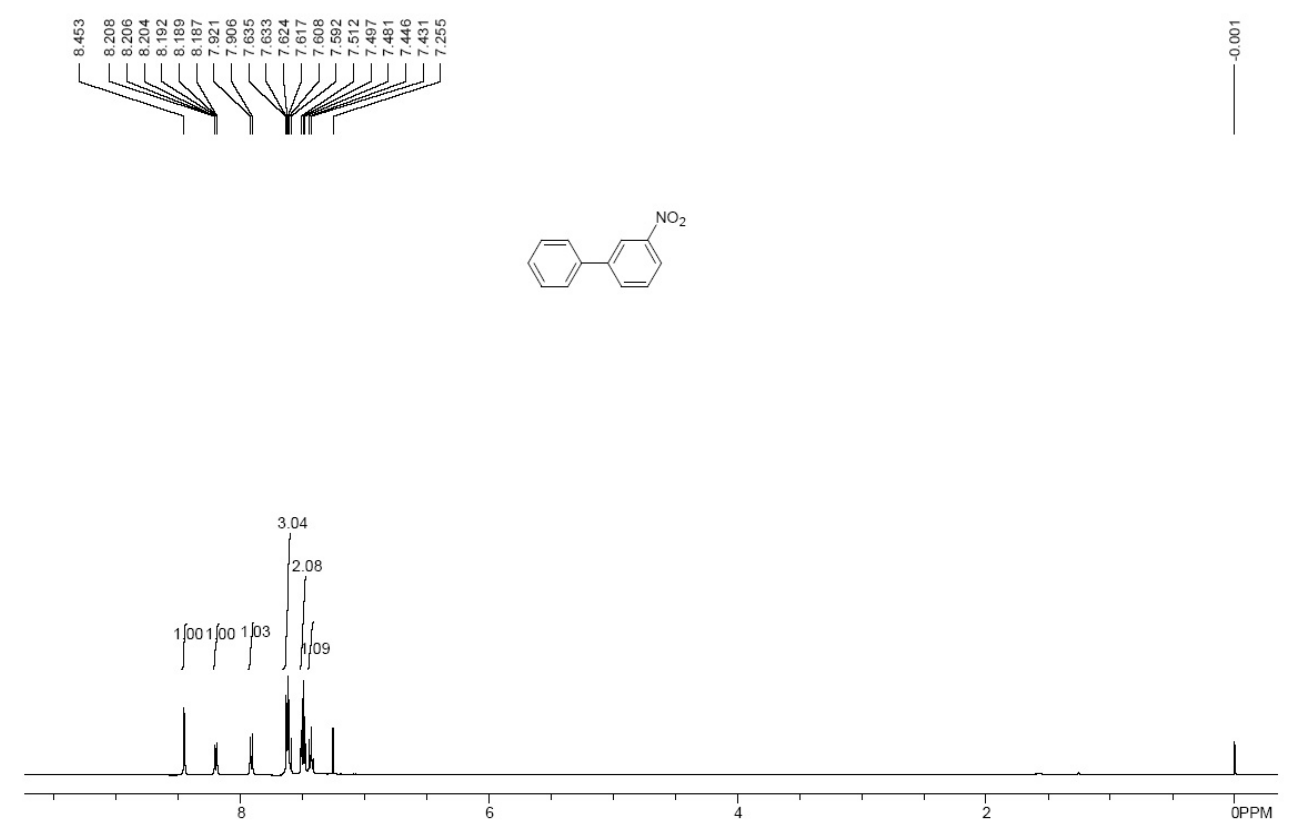


\section{T 3-5 4'-Methyl-3-nitro-biphenyl [53812-68-3, ref 10]}

${ }^{1} \mathrm{H}$ NMR $\left(500 \mathrm{MHz}, \mathrm{CDCl}_{3}, \mathrm{TMS}\right) \delta 8.43(\mathrm{t}, 1 \mathrm{H}, J=2.0 \mathrm{~Hz}), 8.16(\mathrm{~m}, 1 \mathrm{H}), 7.89(\mathrm{q}, 1$ $\mathrm{H}, J=1.5 \mathrm{~Hz}), 7.58(\mathrm{t}, 1 \mathrm{H}, J=7.5 \mathrm{~Hz}), 7.52(\mathrm{~d}, 2 \mathrm{H}, J=8.0 \mathrm{~Hz}), 7.29(\mathrm{~d}, 2 \mathrm{H}, J=8.0$

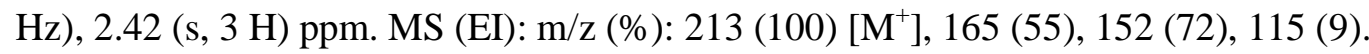
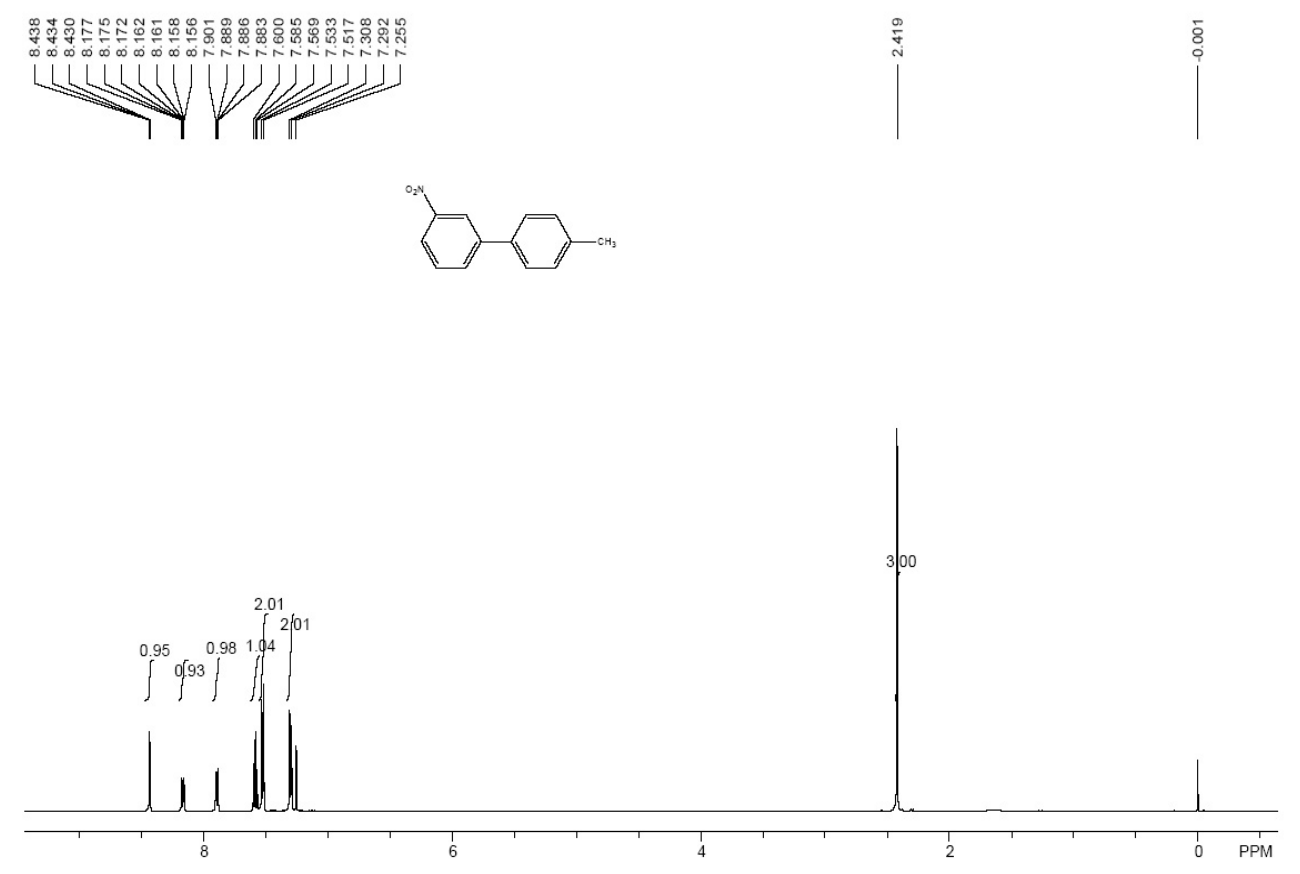
T 3-6 3-Cyano-biphenyl [24973-50-0, ref 10]

${ }^{1} \mathrm{H}$ NMR (500 MHz, $\mathrm{CDCl}_{3}$, TMS) $\delta 7.82(\mathrm{~d}, 1 \mathrm{H}, J=1.5 \mathrm{~Hz}), 7.79(\mathrm{~m}, 1 \mathrm{H}), 7.60(\mathrm{~m}, 1$

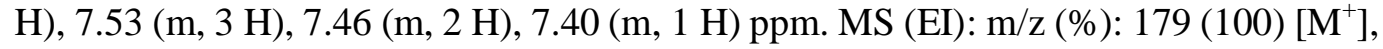
$153(9), 115$ (17).
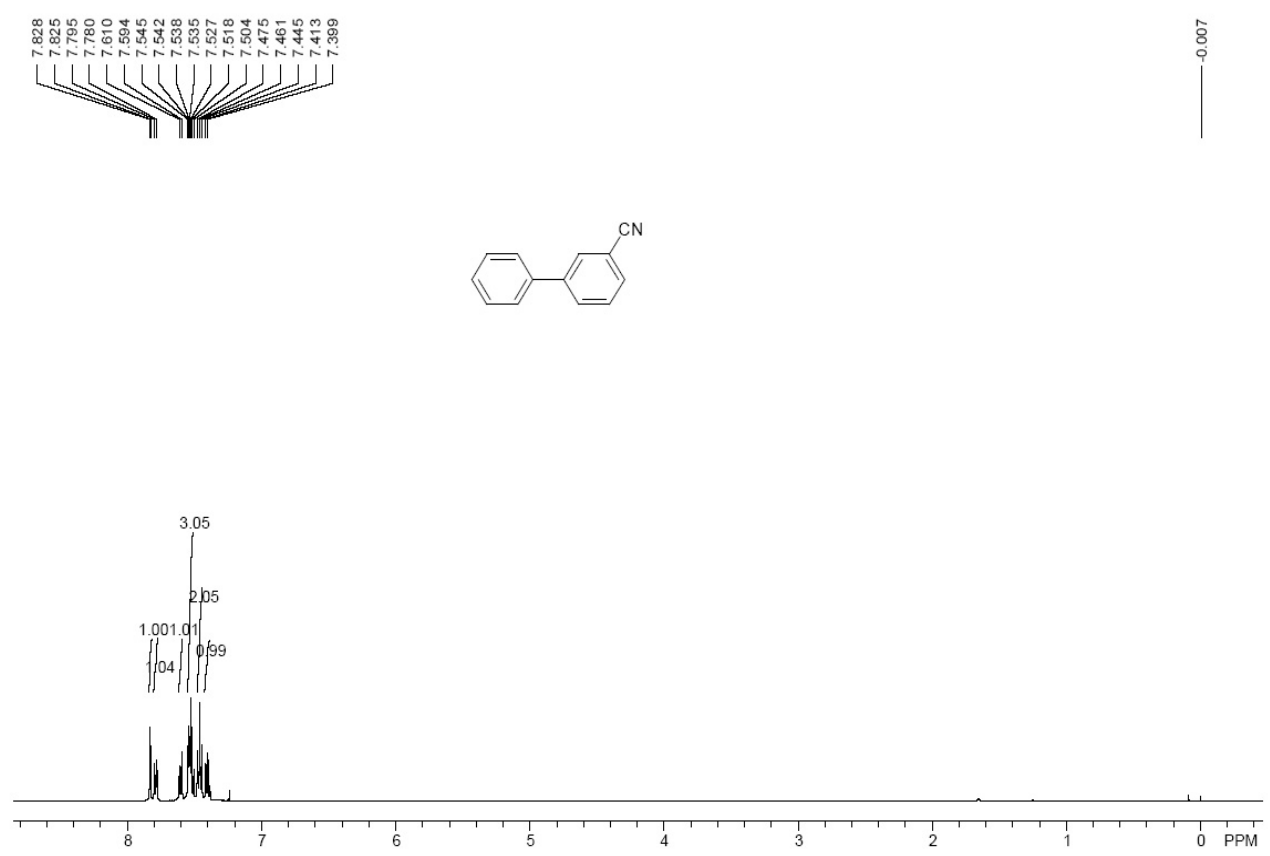
T 3-7 4'-Methoxy-3-cyano-biphenyl [154197-00-9, ref 10]

${ }^{1} \mathrm{H}$ NMR (500 MHz, CDCl 3 , TMS) $\delta 7.78(\mathrm{~s}, 1 \mathrm{H}), 7.74(\mathrm{~d}, 1 \mathrm{H}, J=8.0 \mathrm{~Hz}), 7.54$ (d, 1 $\mathrm{H}, J=7.5 \mathrm{~Hz}), 7.47(\mathrm{t}, 3 \mathrm{H}, J=4.0 \mathrm{~Hz}), 6.98(\mathrm{~d}, 2 \mathrm{H}, J=9.0 \mathrm{~Hz}), 3.84(\mathrm{~s}, 3 \mathrm{H}) \mathrm{ppm}$. MS (EI): m/z (\%): 209 (100) [M+], 194 (43), 166 (51), 140 (31).
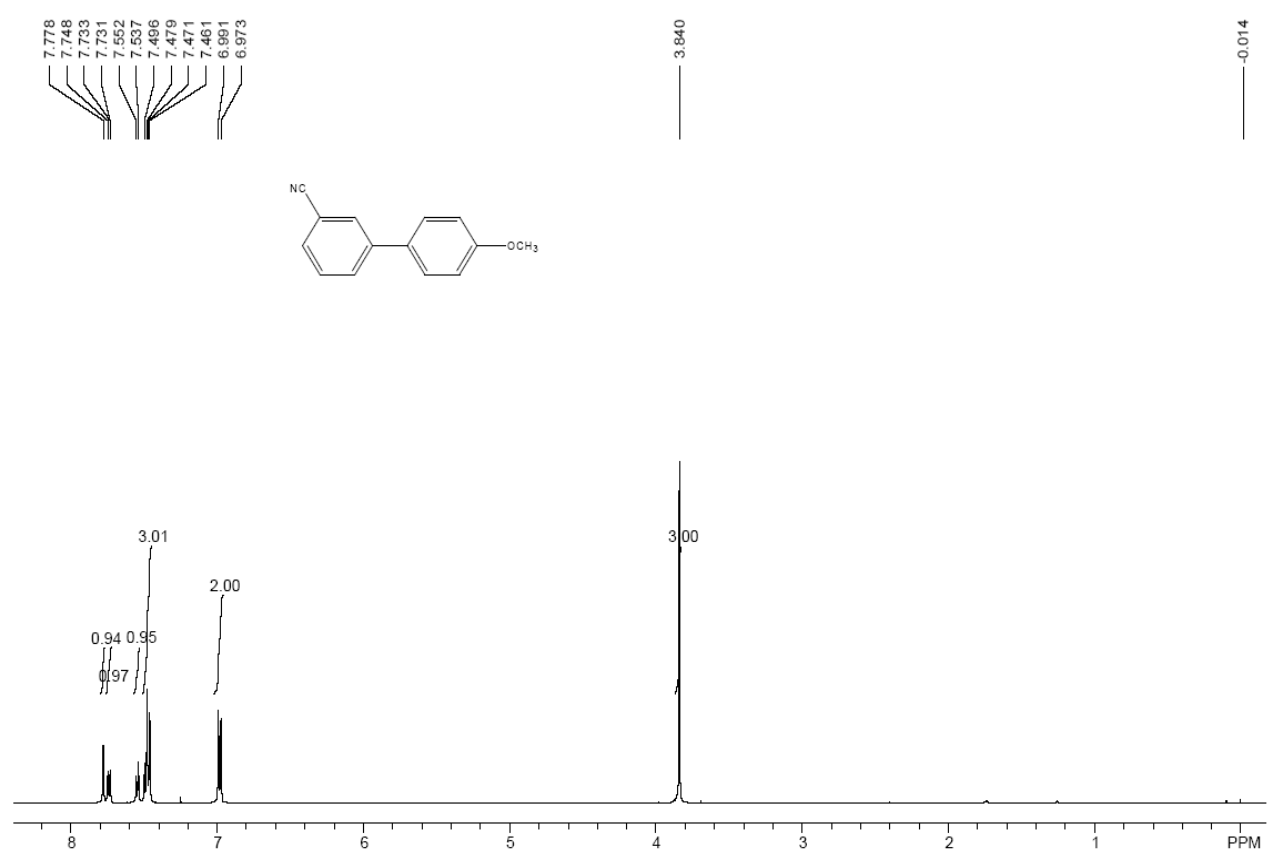
T 3-8 2-Nitro-biphenyl [86-00-0, ref 10]

${ }^{1} \mathrm{H}$ NMR (500 MHz, $\left.\mathrm{CDCl}_{3}, \mathrm{TMS}\right) \delta 7.84(\mathrm{q}, 1 \mathrm{H}, J=2.5 \mathrm{~Hz}), 7.60(\mathrm{~m}, 1 \mathrm{H}), 7.44(\mathrm{~m}, 5$

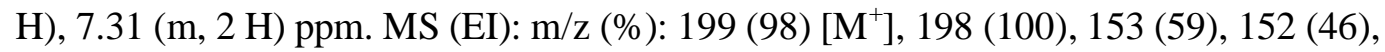

$141(8), 139(8), 115(14), 83(14), 63$ (6), 51 (4).
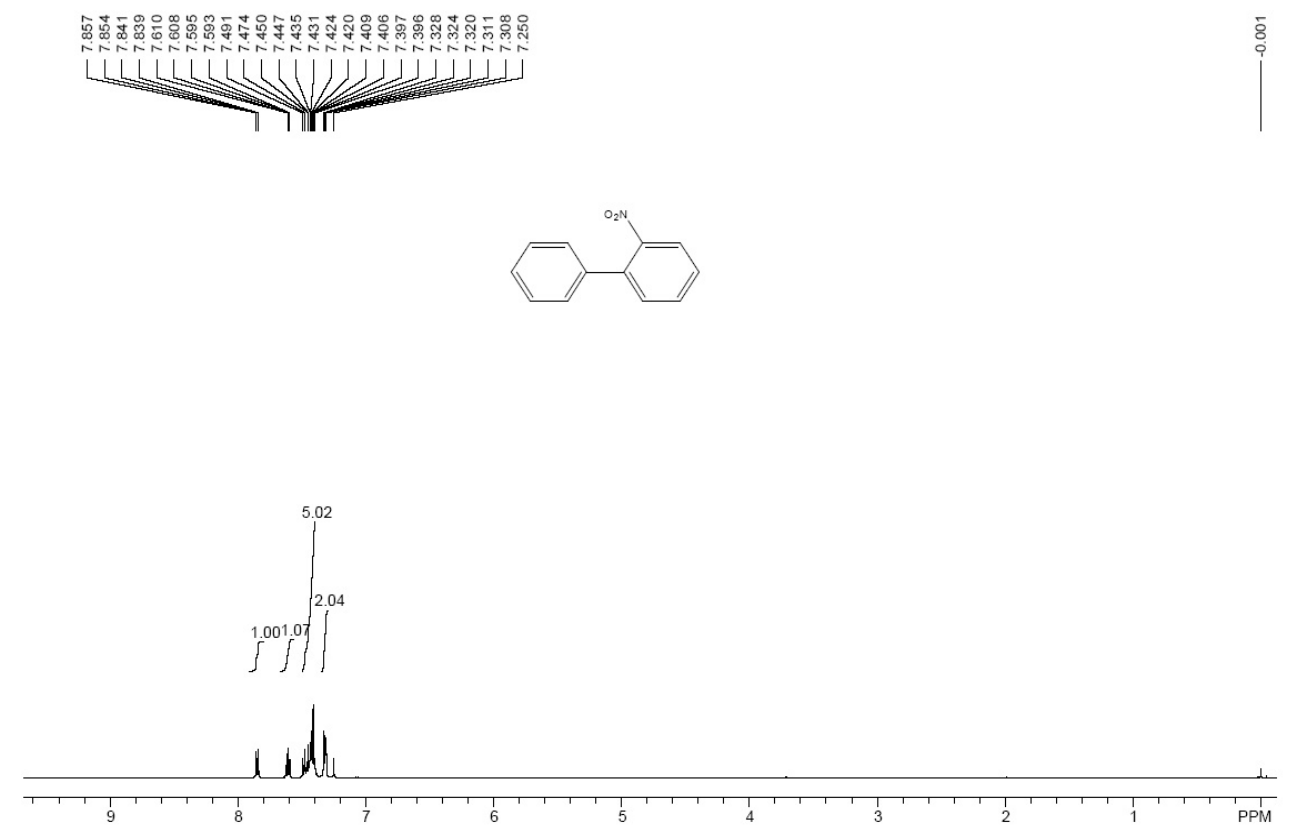
T 3-9 4'-Methyl-2-nitro-biphenyl [20013-55-2, ref 10]

${ }^{1} \mathrm{H}$ NMR (500 MHz, $\mathrm{CDCl}_{3}$, TMS) $\delta 7.84(\mathrm{q}, 1 \mathrm{H}, J=2.5 \mathrm{~Hz}), 7.59(\mathrm{t}, 1 \mathrm{H}, J=2.5 \mathrm{~Hz})$, 7.45 (m, 2 H), 7.23 (m, 4 H), 2.42 (s, 3 H) ppm. MS (EI): m/z (\%): 213 (100) [M+], 198 (20), $196(16), 152(8), 150(8), 115(9)$.
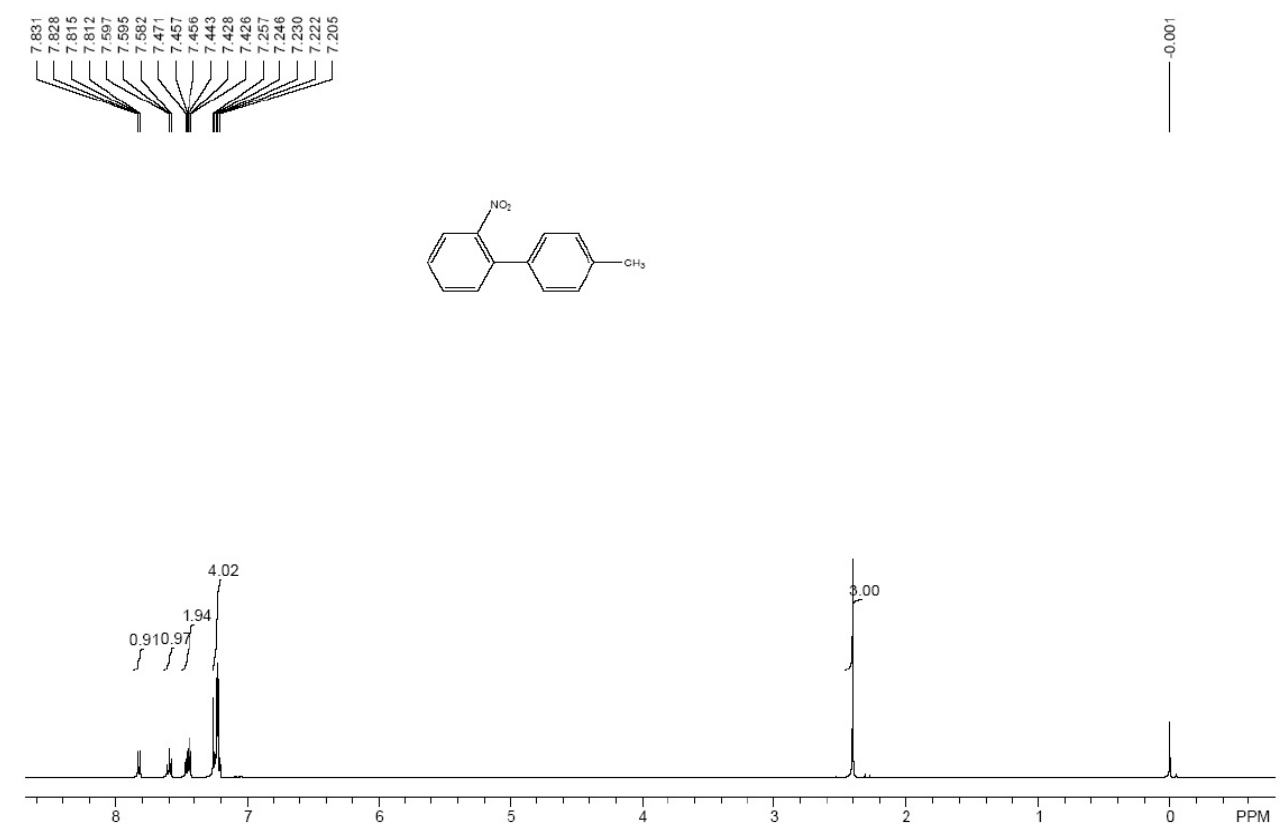
T 3-10 4'-Methoxy-2-nitro-biphenyl [53059-31-7, ref 10]

${ }^{1} \mathrm{H}$ NMR $\left(500 \mathrm{MHz}, \mathrm{CDCl}_{3}, \mathrm{TMS}\right) \delta 7.79(\mathrm{t}, 1 \mathrm{H}, J=3.0 \mathrm{~Hz}), 7.56(\mathrm{q}, 1 \mathrm{H}, J=3.0 \mathrm{~Hz})$, $7.42(\mathrm{t}, 2 \mathrm{H}, J=5.0 \mathrm{~Hz}), 7.25(\mathrm{~m}, 2 \mathrm{H}), 6.95(\mathrm{q}, 2 \mathrm{H}, J=3.0 \mathrm{~Hz}), 3.80(\mathrm{~s}, 3 \mathrm{H}) \mathrm{ppm} . \mathrm{MS}$ (EI): m/z (\%): 229 (100) [M+', 214 (22), 198 (19), 152 (21), 150 (21), 128 (12), 115 $(9)$.
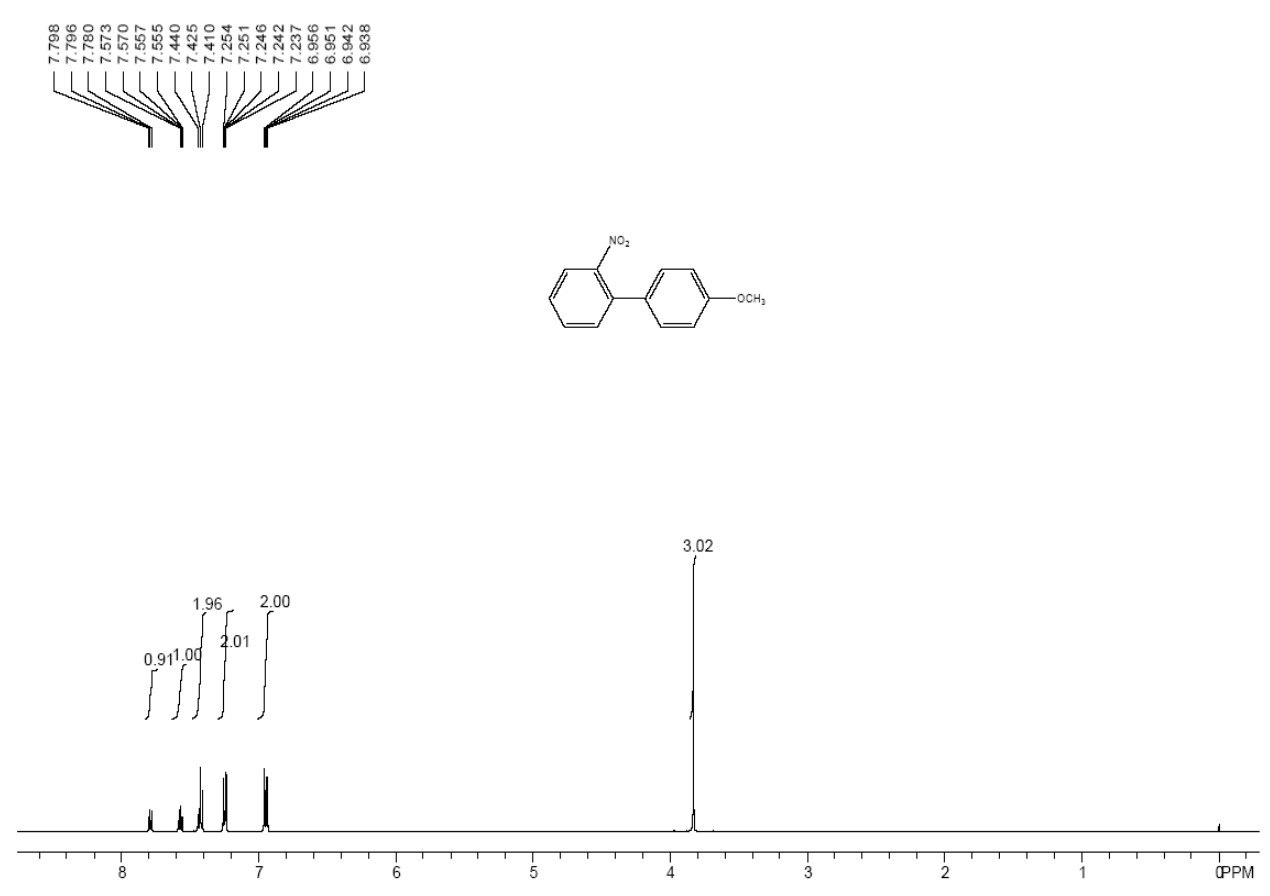
T 3-11 2-Phenyl-pyridine [1008-89-5, ref 11]

${ }^{1} \mathrm{H}$ NMR (500 MHz, $\left.\mathrm{CDCl}_{3}, \mathrm{TMS}\right) \delta 8.70(\mathrm{~d}, 1 \mathrm{H}, J=4.5 \mathrm{~Hz}), 7.98(\mathrm{~d}, 2 \mathrm{H}, J=7.5 \mathrm{~Hz})$, $7.75(\mathrm{~m}, 2 \mathrm{H}), 7.48(\mathrm{t}, 2 \mathrm{H}, J=5.0 \mathrm{~Hz}), 7.42(\mathrm{~d}, 1 \mathrm{H}, J=7.5 \mathrm{~Hz}), 7.24(\mathrm{~d}, 1 \mathrm{H}, J=5.5$

Hz) ppm. MS (EI): m/z (\%): 155 (100) [M+], 154 (21), 153 (7), 115 (7), 76 (9).
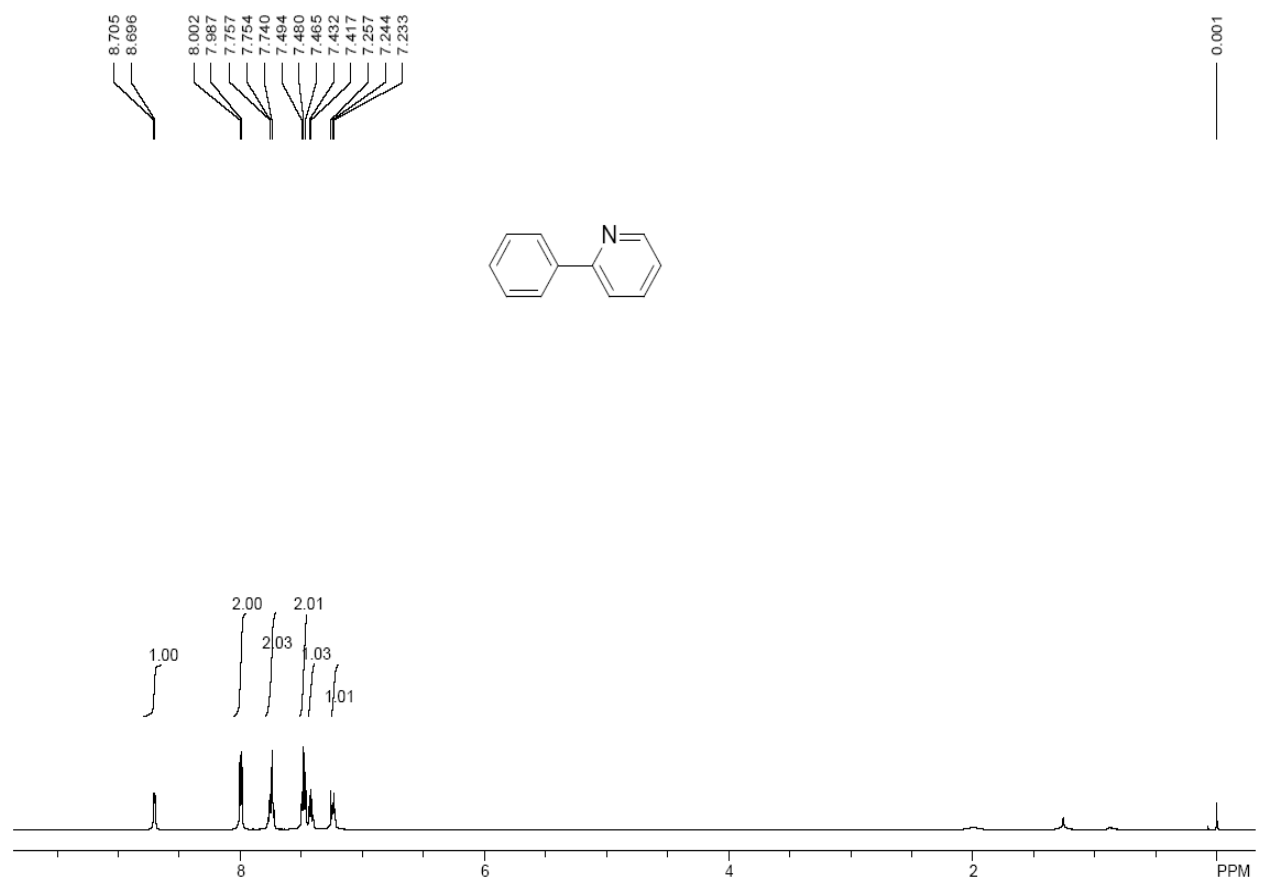
T 3-12 2-(4-Methyl-phenyl)-pyridine [5324-53-8, ref 12]

${ }^{1} \mathrm{H}$ NMR (500 MHz, $\left.\mathrm{CDCl}_{3}, \mathrm{TMS}\right) \delta 8.66(\mathrm{q}, 1 \mathrm{H}, J=2.0 \mathrm{~Hz}), 7.89(\mathrm{~d}, 2 \mathrm{H}, J=8.0 \mathrm{~Hz})$, $7.70(\mathrm{~m}, 2 \mathrm{H}), 7.26$ (t, $2 \mathrm{H}, J=8.0 \mathrm{~Hz}), 7.18$ (m, $1 \mathrm{H}), 2.40$ (s, $3 \mathrm{H}) \mathrm{ppm} . \mathrm{MS}(\mathrm{EI}): \mathrm{m} / \mathrm{z}$ (\%): $169(100)\left[\mathrm{M}^{+}\right], 154(32), 76(5)$
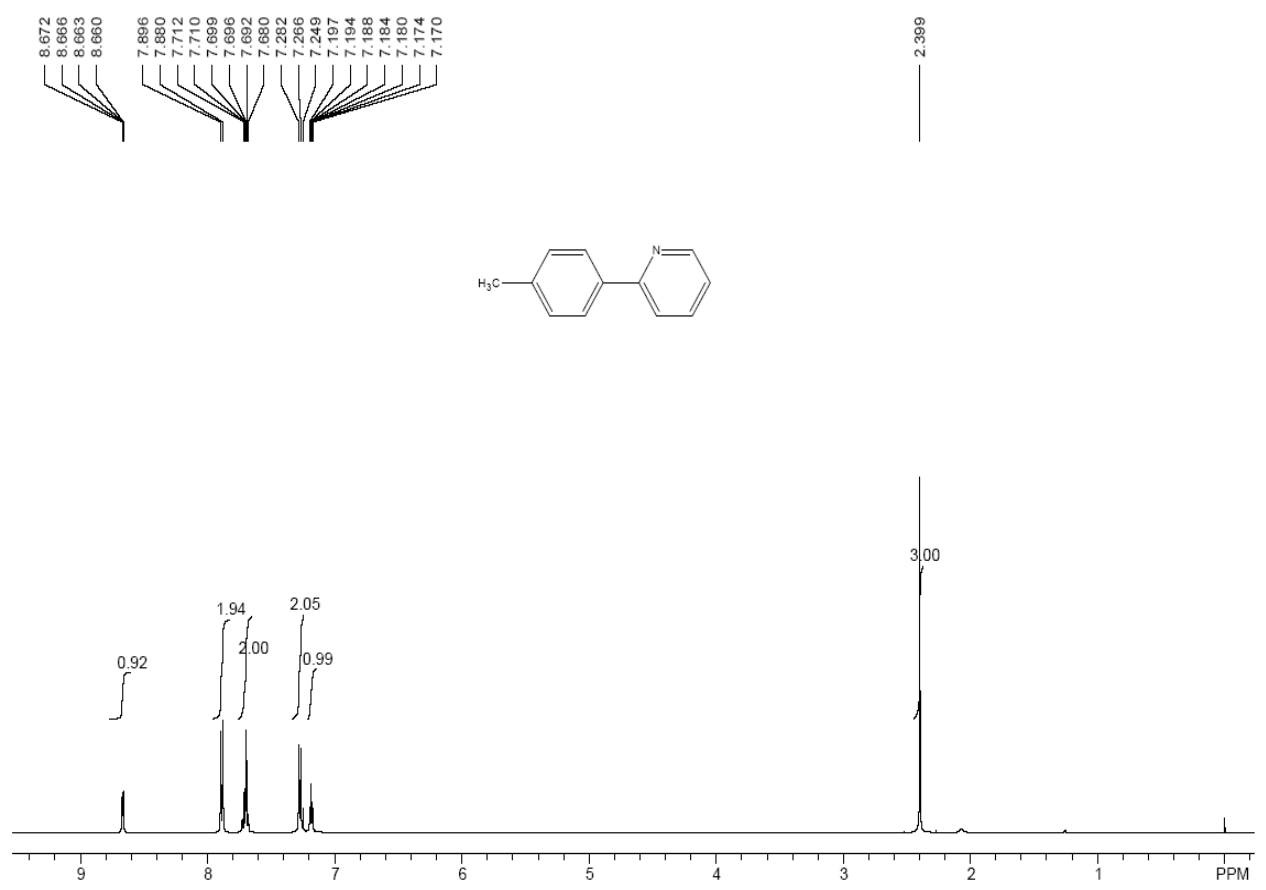
T 3-13 3-Phenyl-pyridine [1008-88-4, ref 13]

${ }^{1} \mathrm{H}$ NMR (500 MHz, $\left.\mathrm{CDCl}_{3}, \mathrm{TMS}\right) \delta 8.85(\mathrm{~d}, 1 \mathrm{H}, J=2.0 \mathrm{~Hz}), 8.59$ (q, $\left.1 \mathrm{H}, J=2.0 \mathrm{~Hz}\right)$, 7.86 (m, 1 H), 7.58 (m, 2 H), 7.47 (m, 2 H), 7.41 (m, 2 H) ppm. MS (EI): m/z (\%): 155 (100) $\left[\mathrm{M}^{+}\right], 154(22), 153(15), 76(9)$
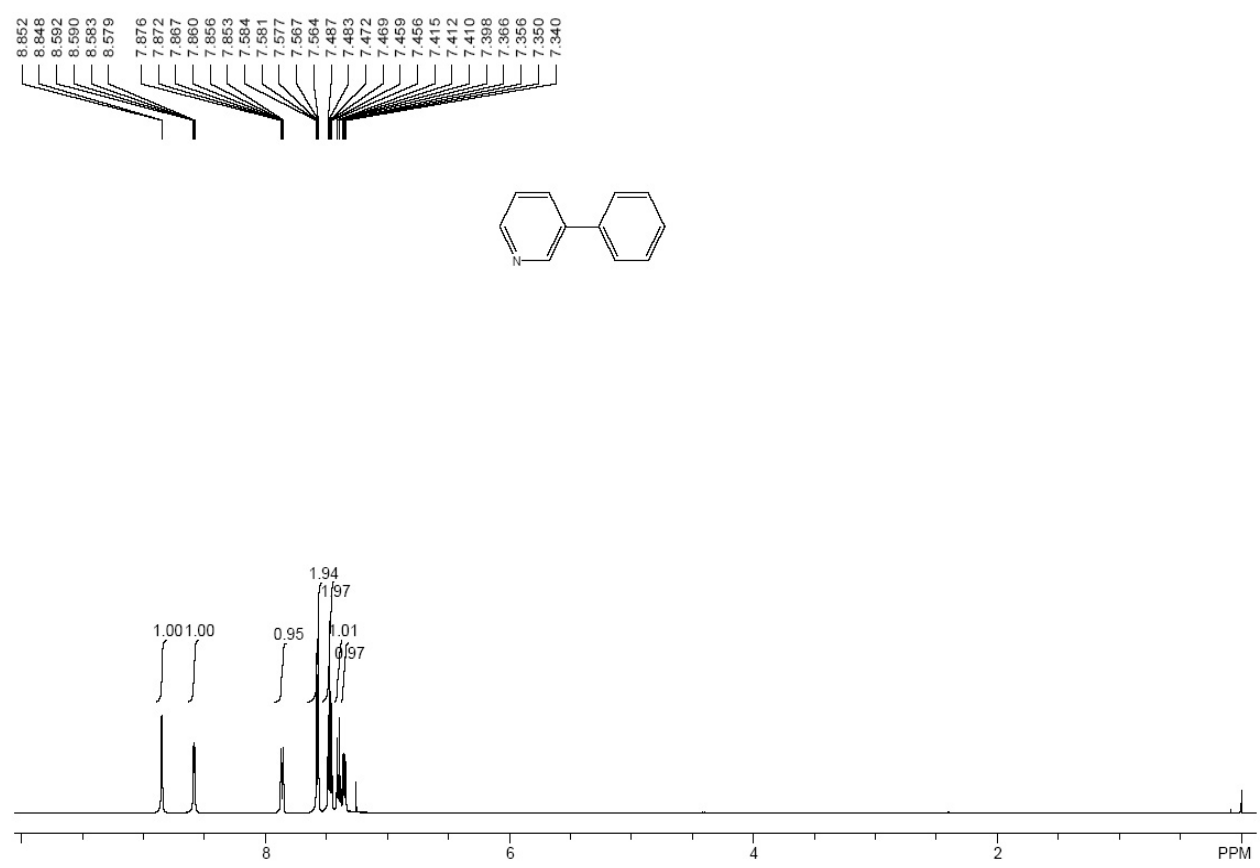
T 3-14 3-(4-Methyl-phenyl)-pyridine [1008-88-4, ref 13]

${ }^{1} \mathrm{H}$ NMR $\left(500 \mathrm{MHz}, \mathrm{CDCl}_{3}\right.$, TMS) $\delta 8.84(\mathrm{~s}, 1 \mathrm{H}), 8.56(\mathrm{~d}, 1 \mathrm{H}, J=4.0 \mathrm{~Hz}), 7.87(\mathrm{~m}, 1$ H), $7.48(\mathrm{~d}, 2 \mathrm{H}, J=7.5 \mathrm{~Hz}), 7.36(\mathrm{~m}, 1 \mathrm{H}), 7.29(\mathrm{~d}, 2 \mathrm{H}, J=7.5 \mathrm{~Hz}), 2.41(\mathrm{~s}, 3 \mathrm{H}) \mathrm{ppm}$. MS (EI): m/z (\%): 169 (100) [M+], 154 (22), 153 (15), 76 (9).
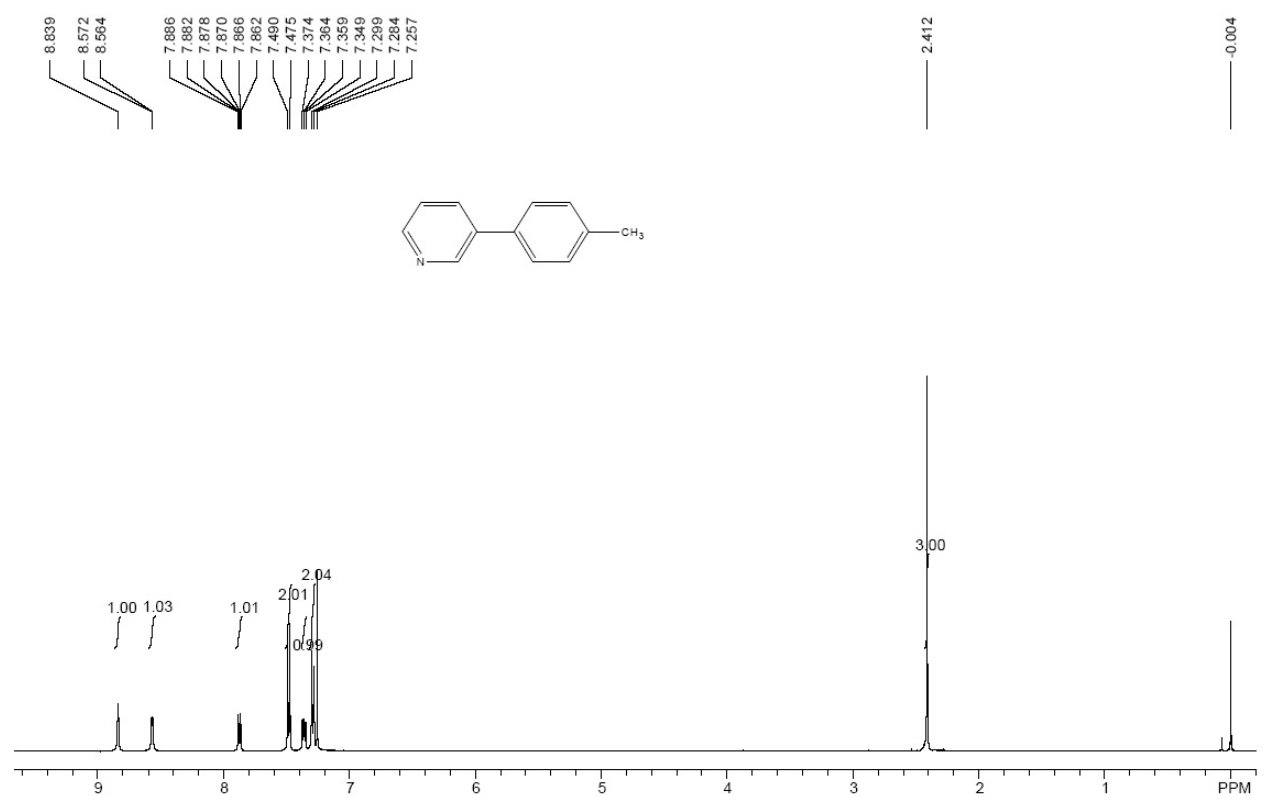
T 3-15 2-(4-Methoxy-phenyl)- thiophene [42545-43-7, ref 14]

${ }^{1} \mathrm{H}$ NMR (500 MHz, $\mathrm{CDCl}_{3}$, TMS) $\delta 7.53$ (q, $\left.2 \mathrm{H}, J=3.0 \mathrm{~Hz}\right), 7.20(\mathrm{~m}, 2 \mathrm{H}), 7.04(\mathrm{~m}, 1$ H), 6.91 (q, $2 \mathrm{H}, J=3.0 \mathrm{~Hz}), 3.83$ (s, $3 \mathrm{H}) \mathrm{ppm} . \mathrm{MS}(\mathrm{EI}): \mathrm{m} / \mathrm{z}(\%): 190(100)\left[\mathrm{M}^{+}\right], 175$ (16), 159 (12), $76(5)$.
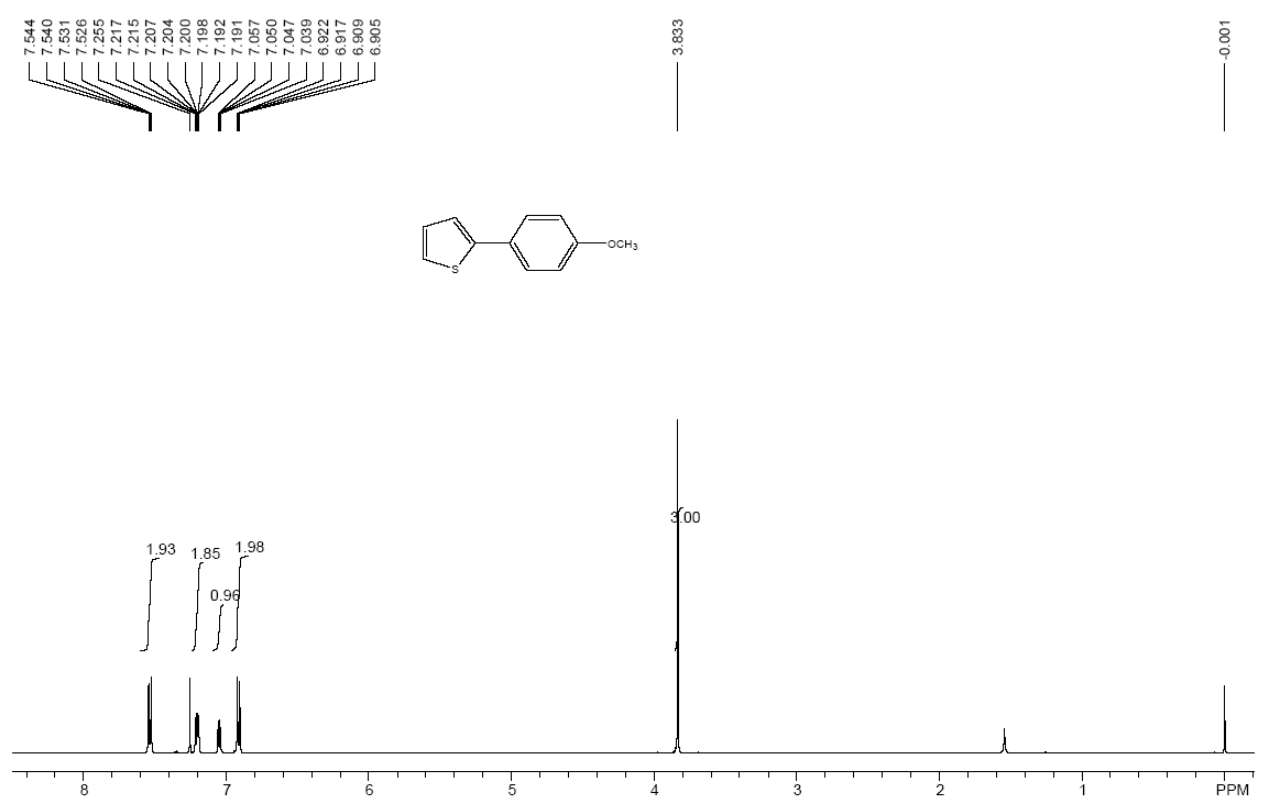
T 3-16 3-(4-Methoxy-phenyl)- thiophene [82437-75-0, ref 15]

${ }^{1} \mathrm{H}$ NMR (500 MHz, $\mathrm{CDCl}_{3}$, TMS) $\delta 7.52(\mathrm{~d}, 2 \mathrm{H}, J=8.5 \mathrm{~Hz}), 7.34(\mathrm{t}, 3 \mathrm{H}, J=5.0 \mathrm{~Hz})$, 6.93 (d, 2 H, $J=8.5 \mathrm{~Hz}), 3.83$ (s, $3 \mathrm{H})$ ppm. MS (EI): m/z (\%): $190(100)\left[\mathrm{M}^{+}\right], 189$ (5), 175 (12), 159 (15), $76(9)$.
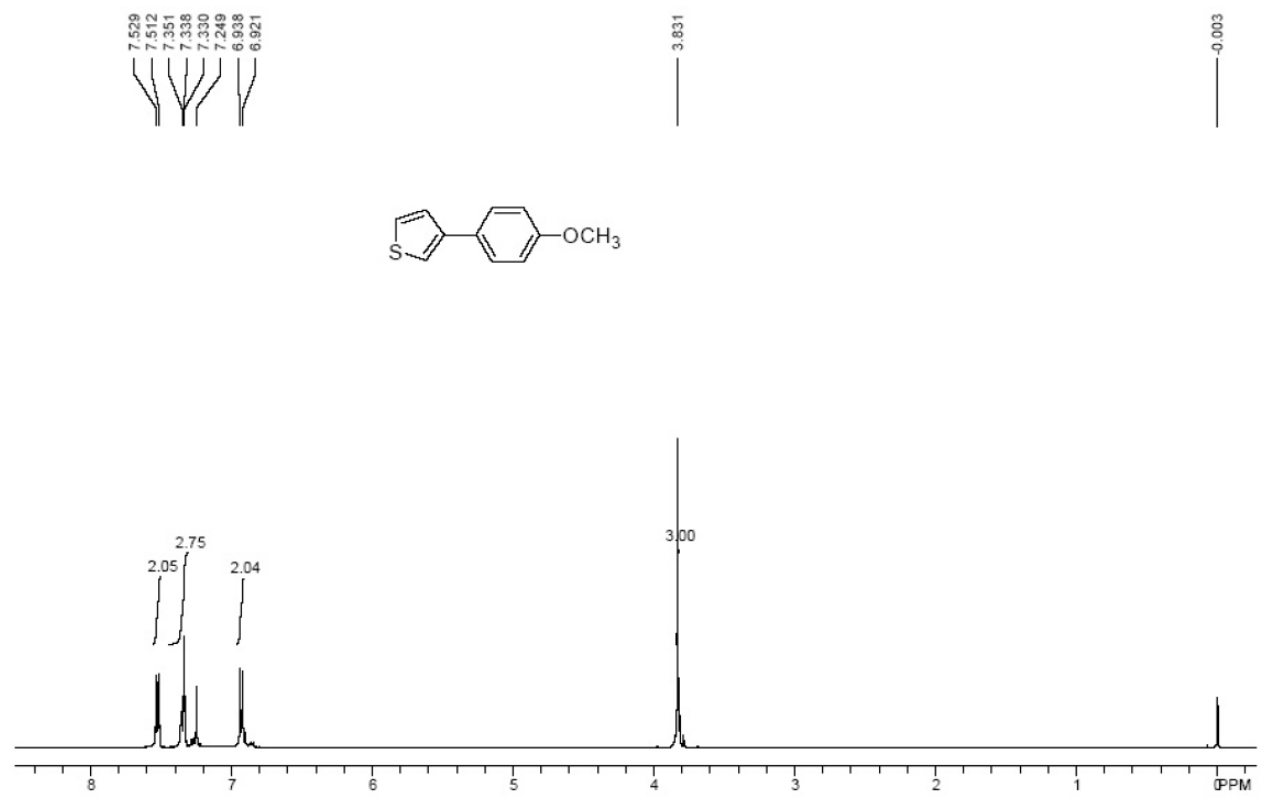

1. Lee, P. H.; Seomoon, D.; Lee, K. Org. Lett. 2005, 7, 343. 
2. Kuroboshi, M.; Waki, Y.; Tanaka, H. J. Org. Chem. 2003, 68, 3938.

3. Li, J.-H.; Xie, Y.-X.; Yin, D.-L. J. Org. Chem. 2003, 68, 9867.

4. Nising, C. F.; Schmid, U. K.; Nieger, M.; Brase, S. J. Org. Chem. 2004, 69, 6830.

5. Hennings, D. D.; Iwama, T.; Rawal, V. H. Org. Lett. 1999, 1, 1205.

6. Atsushi, I.; Kazuya, K.; Hiroshi, S.; Koichiro, O. Tetrahedron. 2000, 56, 9601.

7. Yoshinao, T.; Yoshimi, Y.; Zen-ichi, Y. Tetrahedron. 1979, 35, 329.

8. Tao, B.; Boykin, D.W. J. Org. Chem. 2004, 69, 4330.

9. Kataoka, N.; Shelby, Q.; Stambuli, J. P.; Hartwig, J. F. J. Org. Chem. 2002, 67, 5553.

10. Hassan, J.; Hathroubi, C.; Gozzi, C.; Lemaire, M. Tetrahedron 2001, 57, 7845.

11. Adjabeng, G.; Brenstrum, T.; Wilson, J.; Frampton, C.; Robertson, A.; Hillhouse, J.; McNulty, J.; Capretta, A. Org. Lett. 2003, 5, 953.

12. Corinne, G.; Sarah, L.; Jean-Yves, N.; Jacques, P. Tetrahedron 1998, 54, 1289.

13. Le Notre, J.; Firet, J. J.; Sliedregt, L. A. J. M.; van Steen, B. J.; van Koten, G.; Klein Gebbink, R. J. M. Org. Lett. 2005, 7, 363.

14. Deng, Y.; Gong, L.; Mi, A.; Liu, H.; Jiang, Y. Synthesis. 2003, 337.

15. André, G.; Yongxin, H.; Petpiboon, P. Tetrahedron. Lett., 1997, 38, 3841. 